\title{
NASA Trapezoidal Wing Computations Including Transition and Advanced Turbulence Modeling
}

\author{
C. L. Rumsey* and E. M. Lee-Rausch ${ }^{\dagger}$ \\ NASA Langley Research Center, Hampton VA 23681-2199
}

\begin{abstract}
Flow about the NASA Trapezoidal Wing is computed with several turbulence models by using grids from the first High Lift Prediction Workshop in an effort to advance understanding of computational fluid dynamics modeling for this type of flowfield. Transition is accounted for in many of the computations. In particular, a recently-developed 4-equation transition model is utilized and works well overall. Accounting for transition tends to increase lift and decrease moment, which improves the agreement with experiment. Upper surface flap separation is reduced, and agreement with experimental surface pressures and velocity profiles is improved. The predicted shape of wakes from upstream elements is strongly influenced by grid resolution in regions above the main and flap elements. Turbulence model enhancements to account for rotation and curvature have the general effect of increasing lift and improving the resolution of the wing tip vortex as it convects downstream. However, none of the models improve the prediction of surface pressures near the wing tip, where more grid resolution is needed.
\end{abstract}

\section{Nomenclature}

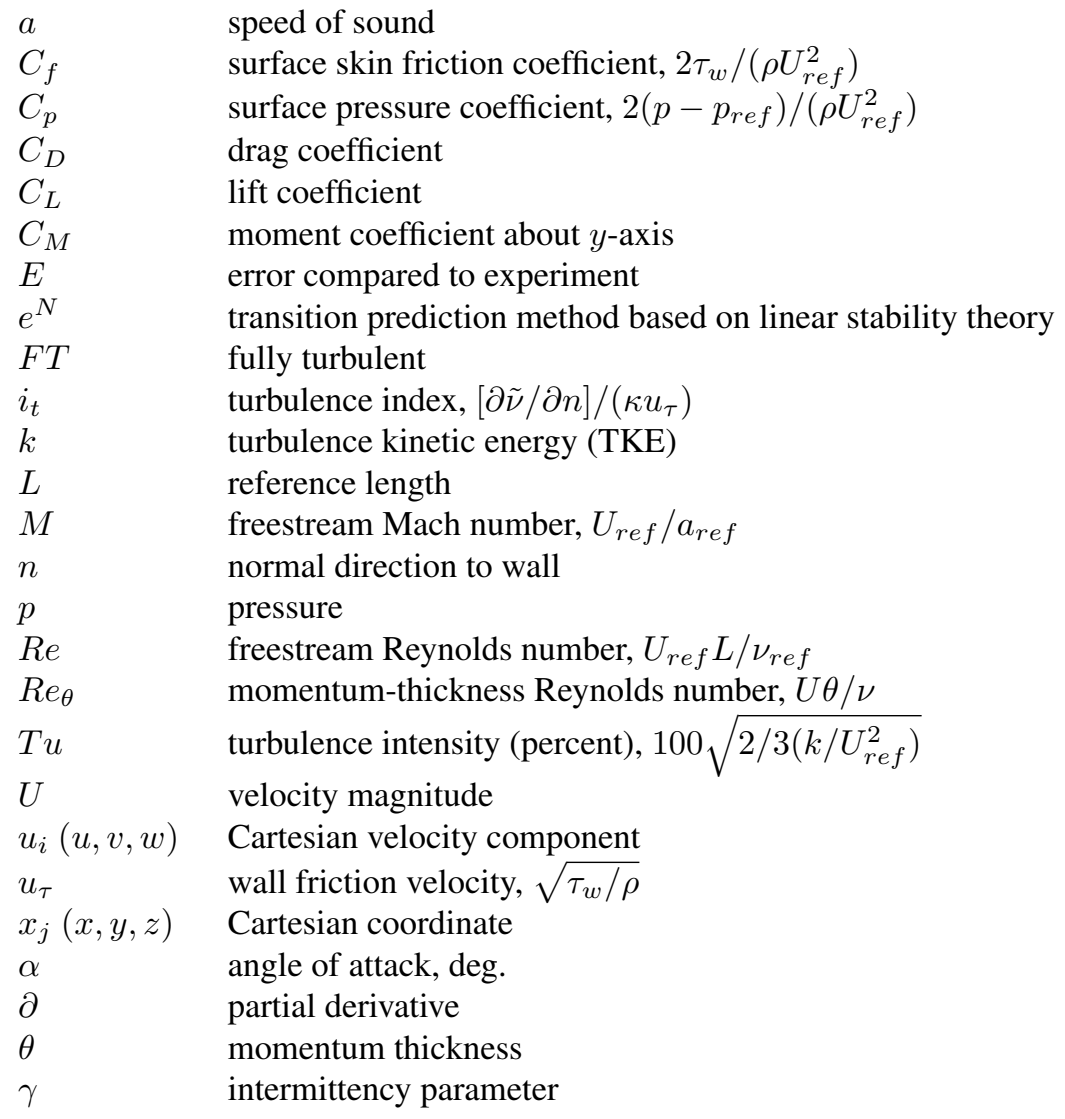

\footnotetext{
* Senior Research Scientist, Computational AeroSciences Branch, C.L.Rumsey@ nasa.gov. Fellow AIAA.

${ }^{\dagger}$ Research Engineer, Computational AeroSciences Branch, E.Lee-Rausch@nasa.gov. Senior Member AIAA.
} 


$\begin{array}{ll}\kappa & \text { Von Karman law-of-the-wall constant, } 0.41 \\ \mu_{t} & \text { turbulent eddy viscosity } \\ \rho & \text { density } \\ \nu & \text { kinematic viscosity } \\ \tilde{\nu} & \text { turbulent variable from Spalart-Allmaras model } \\ \tau_{i j} & \text { turbulent shear stress tensor } \\ \tau_{w} & \text { wall shear stress } \\ \Omega & \text { vorticity magnitude } \\ \text { Subscript } & \\ \text { max } & \text { maximum } \\ r e f & \text { reference conditions (free stream) } \\ x & \text { based on } x \text {-distance }\end{array}$

\section{Introduction}

The first AIAA High Lift Prediction Workshop (HiLiftPW-1) established a baseline for current state-of-the-art computational fluid dynamics (CFD) code capabilities on high-lift configurations. ${ }^{1,2}$ At the workshop, ${ }^{\text {a }}$ different research groups using a variety of codes and methods studied the 3 -element NASA Trapezoidal Wing configuration. ${ }^{3,4}$ Particular assessments were made for fully turbulent flow with two different flap settings. The importance of transition was known, but the first workshop's cases were limited to fully turbulent flow to attain more useful comparisons between a large number of codes. As a whole, the collective CFD results on a clean configuration (no slat or flap support brackets modeled) tended to under-predict the lift and the magnitude of the pitching moment compared to experiment. However, the trend with grid refinement was generally in the right direction (i.e., finer grids produced higher lift). Including slat and flap support brackets in the CFD geometry tended to lower the predicted lift. Agreement (both between different CFD results as well as between CFD and experiment) deteriorated at higher angles of attack approaching stall. The outboard region of the wing was the most problematic area for CFD, with the least consistency among CFD codes, and the largest deviations from experiment.

There were many publications of CFD results after the workshop. ${ }^{5-18}$ Most of these studies involved a few wellknown "standard" turbulence models such as Spalart-Allmaras (SA) ${ }^{19}$ and Menter's shear-stress transport (SST) ${ }^{20}$ applied in fully turbulent mode. At the workshop, SA (fully turbulent) was heavily favored because it tended to yield closer agreement to experiment for the Trapezoidal Wing. SST tended to separate more readily than SA and consequently produced lower lift. However, it was noted at the workshop that transition effects could alter this trend. Transition tended to reduce trailing edge flap separation and significantly improve pitching moment predictions. Subsequently, the papers of Steed, ${ }^{6}$ Fares and Nolting, ${ }^{11}$ and Eliasson et al. ${ }^{9,17}$ explored the important effects of including transition in the CFD simulations. Steed used the 4-equation $\gamma-R e_{\theta}$ SST transition/turbulence model of Langtry and Menter. ${ }^{21}$ This model includes the solution of two additional transport equations for an intermittency parameter and a local transition onset momentum-thickness Reynolds number. The effective intermittency regulates the production of turbulence kinetic energy (TKE) in the SST model. Fares and Nolting fixed laminar regions in a lattice Boltzmann solver based on measurements described by McGinley et al. ${ }^{22}$ Eliasson et al. used a database $e^{N}$ method with the envelope approach to impose transition regions in conjunction with the SA model. All groups demonstrated improved results when transition regions were included. The importance of including transition for multi-element airfoil computations was similarly shown to be very important for two-dimensional flows over a decade ago. ${ }^{23}$

In the current validation study, some of the best practices from the earlier studies mentioned above are applied to the Trapezoidal Wing in an effort to advance our understanding of high lift flowfields. Many cases are included, using different codes, grids, and turbulence models. Through particular cases in the matrix of runs, an attempt is made to demonstrate both isolated and combined effects. The study includes the $\gamma-R e_{\theta}$ SST transition/turbulence model of Langtry and Menter, ${ }^{21}$ and we also investigate setting transition locations in an a priori fashion. Both structured- and unstructured-grid solvers are employed, and results are examined both with and without support brackets included.

The influences of rotation and curvature corrections to turbulence models are also investigated. These could be influential for regions of the flow such as near the wing tip where a strong vortex forms and subsequently convects downstream. Turbulence model variations tested include the three rotation and curvature-corrected turbulence models SA-R, ${ }^{24}$ SA-RC ${ }^{25}$ and SST-RC. ${ }^{26,27}$ In particular, the ability of these models to resolve the Trap Wing's tip vortex are

${ }^{a}$ http://hiliftpw.larc.nasa.gov; cited 5/20/2012. 
assessed compared to the baseline SA and SST models.

\section{Methods and Models}

Two CFD codes are employed in this study. CFL3D is a cell-centered, structured-grid, upwind-biased Reynoldsaveraged Navier-Stokes (RANS) code, ${ }^{28}$ and FUN3D is a node-centered, unstructured-grid, upwind-biased RANS code. ${ }^{29}$ These codes were also used in an earlier study associated with HiLiftPW-1. ${ }^{12}$ Transition is accounted for in two different ways: (1) by zeroing out turbulence production terms in laminar regions on each of the elements by using regions specified by Eliasson et al. ${ }^{17}$ (also provided on the HiLiftPW website), and (2) by employing the 4-equation $\gamma-R e_{\theta}$ SST transition/turbulence model of Langtry and Menter. ${ }^{21}$

In CFL3D and FUN3D, the default implementations of the production terms for the SST and $\gamma-\operatorname{Re}_{\theta}$ SST models make use of the "vorticity approximation," introduced by Menter ${ }^{30}$ and referred to elsewhere ${ }^{\mathrm{b}}$ as "SST-V." In this approximation, the production term $\tau_{i j} \partial u_{i} / \partial x_{j}$ is replaced with $\mu_{t} \Omega^{2}$, where $\mu_{t}$ is the eddy viscosity and $\Omega$ is the magnitude of vorticity. This approximation has been shown to be reasonable for many aerodynamic flows, and it avoids over-production of TKE through strong shocks and near stagnation points that occurs with the standard strain production form. A separate run of the $\gamma-R e_{\theta}$ SST model was made with CFL3D using the standard strain production form, and it made a dramatic difference (4\% decrease in lift, 5\% decrease in drag, and $7 \%$ increase in moment) compared to the use of the vorticity production form. With the standard strain production form, the tendency of overproduction of TKE near stagnation points adversely influences the 4-equation model's attempt to laminarize near the leading edges of the main and flap elements. Note that there are other production term limiters (Langtry ${ }^{31}$ ), modified production term forms (Kato and Launder ${ }^{32}$ ), and time scale limiters $\left(\operatorname{Durbin}^{33}\right.$ ) that also improve stagnation region behavior. All results shown below use the vorticity approximation ("SST-V").

Several rotation and curvature corrections to the SA and SST models were investigated. The SA-R model ${ }^{24}$ reduces the eddy viscosity in regions where vorticity exceeds strain rate, such as in vortex core regions where pure rotation should not produce turbulence. The SA-RC model ${ }^{25}$ accounts for both rotation and curvature effects in the SA model through a function of the Lagrangian derivative of the strain rate tensor. The SST-RC model ${ }^{26,27}$ sensitizes the SST model to curvature based on an approximation to the gradient Richardson number. Note that for SA-RC, the constant $c_{r 3}$ was taken as 0.6 (based on an earlier study ${ }^{34}$ ), and for SST-RC the constant $C_{r c}=1.4$ was used from Mani et al. ${ }^{27}$

As one of several validation exercise for the recent implementation of the $\gamma-R_{\theta}$ SST model in NASA codes, the flat plate was computed using various freestream turbulence intensity and eddy viscosity levels. The cases were taken from Langtry and Menter. ${ }^{21}$ Here, the codes CFL3D, FUN3D, and OVERFLOW ${ }^{35}$ were computed on the same hexahedral grid with plate length of $4.367 L, M=0.2$, and $R e / L=1 \times 10^{6}$. Skin friction results are shown in Fig. 1 on page 11. All three codes yielded similar results to each other, and agreed reasonably well with experiment.

\section{Results}

For the NASA Trapezoidal Wing, computations were performed for configuration 1 (slat at $30^{\circ}$, flap at $25^{\circ}$ ). The $R e$ based on mean aerodynamic chord ( $L=39.634$ inches) was 4.2 million and $M=0.2$. A range of angles of attack between $6^{\circ}$ and $37^{\circ}$ were tested, with a focus on the $\alpha=13^{\circ}$ and $\alpha=28^{\circ}$ cases. Table 1 shows a summary of computations performed for this study. In the $\gamma-R_{\theta}$ SST runs, the far-field turbulence intensity was set to $T u=0.075 \%$ (from NASA Langley 14 - by 22 -foot wind tunnel conditions ${ }^{36}$ ), and far-field eddy viscosity was set to $\mu_{t} / \mu_{\text {ref }}=0.2$. A higher value of $\mu_{t} / \mu_{\text {ref }}=10$ was also tried for one case, and it made little difference. So-called "sustaining terms" ${ }^{37}$ were not employed for the current study, due to uncertainty in their effect on the $\gamma-R_{\theta}$ SST model, which had been calibrated by Langtry and Menter without them. The term "FT" in the table indicates fully turbulent, and the term "trans set" indicates that transition locations were fixed a priori, as described in Section II. Note that some of the listed computations were actually a part of the earlier study by Park et al., ${ }^{12}$ but they are included here for comparison purposes.

To provide some representative results in tabular form, a summary of selected forces and moments for various run combinations of code, grid, turbulence model, and transition treatment at $\alpha=13^{\circ}$ and $28^{\circ}$ is given in Table 2. Note that it proved to be very difficult to run at higher angles of attack such as $\alpha=28^{\circ}$ with transition fixed a priori. The flowfield tended to separate (stall) early and resist convergence. Fixing transition locations was also tedious and required knowing locations prior to running, which is not generally feasible unless coupled to a method such as $e^{N}$. Furthermore, the regions where laminar flow was specified sometimes appeared to retain too-high levels

\footnotetext{
${ }^{\mathrm{b}}$ http://turbmodels.larc.nasa.gov; cited 5/20/2012.
} 
of turbulence (because of turbulence advection from upstream). Various options (such as SA-R) could mitigate the degree of upstream influence, but the process in general was not very straightforward.

Three different grid systems, available on the HiLiftPW website, were employed in this study. All computations were performed on "medium" grid levels from the workshop grid refinement study. Grid study results for the UH6 and SX1 grids can be found in Park et al. ${ }^{12}$ The grids denoted as UH6 (without brackets) and UH16 (both with and without brackets) were unstructured mixed-element grids. SX1 (without brackets) was a structured multi-zone grid, and UX9 was the same grid written in unstructured format (hexahedra). For the medium levels, the mixed-element UH6 grid (no brackets) had 11 million grid points, UH16 (no brackets) had 28 million, UH16 (brackets) had 32.5 million, and SX1/UX9 (no brackets) had 52 million. Additional details about these grids can be found in other references. ${ }^{2,5,13}$ At HiLiftPW-1, including the brackets was found to have a noticeable influence on results. For example, at $\alpha=28^{\circ}$ the lift coefficient decreased by approximately $0.06-0.08$ when brackets were modeled.

Figure 2 on page 11 shows two representative views of the underside of the configuration, one without and one with brackets. Figure 2(a) is the structured SX1 grid, and (b) is the unstructured UH16 grid. The surface grid points on the main element are shown in red. Figure 3 on page 12 shows the wing upper surface, along with locations where pressure taps on the model were located and where 7-hole-probe velocity data were taken. In the current study, surface pressures are compared only at 3 stations on the flap, as depicted in the figure by orange.

Table 1: Summary of computations

\begin{tabular}{lllll}
\hline Code & Grid & Brackets? & Turb Model & Focus \\
\hline FUN3D & UH6 & No & SA (FT) & full polar \\
FUN3D & UH16 & No & SA (FT) & full polar \\
FUN3D & UH16 & Yes & SA (FT) & full polar \\
FUN3D & UH16 & Yes & SA-RC (FT) & limited $\alpha$ \\
FUN3D & UH16 & Yes & SA (trans set) & limited $\alpha$ \\
FUN3D & UH16 & Yes & SA-R (trans set) & limited $\alpha$ \\
FUN3D & UH16 & Yes & SA-RC (trans set) & limited $\alpha$ \\
FUN3D & UH16 & Yes & SST-RC (trans set) & limited $\alpha$ \\
FUN3D & UH16 & Yes & $\gamma-R e_{\theta}$ SST & full polar \\
FUN3D & SX1/UX9 & No & SA (FT) & limited $\alpha$ \\
CFL3D & SX1/UX9 & No & SA (FT) & full polar \\
CFL3D & SX1/UX9 & No & SST (FT) & full polar \\
CFL3D & SX1/UX9 & No & SA-R (FT) & limited $\alpha$ \\
CFL3D & SX1/UX9 & No & SA-RC (FT) & limited $\alpha$ \\
CFL3D & SX1/UX9 & No & $\gamma-R e_{\theta}$ SST & full polar \\
CFL3D & SX1/UX9 & No & $\gamma-R e_{\theta}$ SST-RC & full polar \\
\hline
\end{tabular}

\section{A. Forces and Moments}

Figure 4 on page 13 shows a summary of the computed lift, drag, and pitching moment coefficients for several different representative cases. CFL3D was only run without brackets. As shown in the left-half column (Figs. 4(a), (c), and (e)), for CFL3D the SA model run fully turbulent ("FT") produced very reasonable results compared to experiment, slightly low in lift over most of the lift curve. The SST model run fully turbulent yielded significantly lower lift—especially at high angles of attack - and a higher moment overall. As noted at the workshop, SST tended to produce a greater amount of separated flow than SA on the flap for many workshop participants. On the other hand, the 4-equation $\gamma-R e_{\theta}$ SST model increased the lift and decreased the moment, in excellent agreement with the experiment overall. Below we will explore some detailed results from the $\gamma-R e_{\theta}$ SST model in order to try to understand why this was the case. However, this particular model also resulted in $C_{L, \max }$ that was too high on the SX1 grid. The reasons for this are not known, although including the brackets may be necessary to accurately capture some of the flow physics mechanisms responsible for stall. 
Table 2: Summary of selected forces and moments

\begin{tabular}{|c|c|c|c|c|c|c|c|c|c|}
\hline Code & Grid & Brackets? & Turb Model & $C_{L}$ & $C_{D}$ & $C_{M}$ & $\% E_{C L}$ & $\% E_{C D}$ & $\% E_{C M}$ \\
\hline \multicolumn{10}{|l|}{$\alpha=13^{\circ}$} \\
\hline Experiment & & & & 2.0474 & 0.3332 & -0.5032 & & & \\
\hline FUN3D & UH6 & No & $\mathrm{SA}(\mathrm{FT})$ & 1.9922 & 0.3248 & -0.4707 & -2.7 & -2.5 & -6.5 \\
\hline FUN3D & UH16 & No & SA (FT) & 1.9939 & 0.3254 & -0.4714 & -2.6 & -2.3 & -6.3 \\
\hline FUN3D & UH16 & Yes & $\mathrm{SA}(\mathrm{FT})$ & 1.9801 & 0.3261 & -0.4676 & -3.3 & -2.1 & -7.1 \\
\hline FUN3D & UH16 & Yes & SA-RC (FT) & 1.9886 & 0.3276 & -0.4698 & -2.9 & -1.7 & -6.6 \\
\hline FUN3D & UH16 & Yes & SA (trans set) & 2.0132 & 0.3317 & -0.4812 & -1.7 & -0.5 & -4.4 \\
\hline FUN3D & UH16 & Yes & SA-R (trans set) & 2.0226 & 0.3350 & -0.4857 & -1.2 & 0.5 & -3.5 \\
\hline FUN3D & UH16 & Yes & SA-RC (trans set) & 2.0454 & 0.3354 & -0.4938 & -0.1 & 0.7 & -1.9 \\
\hline FUN3D & UH16 & Yes & SST-RC (trans set) & 2.0271 & 0.3289 & -0.4816 & -1.0 & -1.3 & -4.3 \\
\hline FUN3D & UH16 & Yes & $\gamma-R e_{\theta} \mathrm{SST}$ & 2.0122 & 0.3309 & -0.4797 & -1.7 & -0.7 & -4.7 \\
\hline FUN3D & SX1/UX9 & No & $\mathrm{SA}(\mathrm{FT})$ & 1.9974 & 0.3232 & -0.4771 & -2.4 & -3.0 & -5.2 \\
\hline CFL3D & SX1/UX9 & No & SA (FT) & 2.0165 & 0.3254 & -0.4831 & -1.5 & -2.3 & -4.0 \\
\hline CFL3D & SX1/UX9 & No & SST (FT) & 1.9848 & 0.3180 & -0.4664 & -3.1 & -4.6 & -7.3 \\
\hline CFL3D & SX1/UX9 & No & SA-R (FT) & 2.0295 & 0.3274 & -0.4862 & -0.9 & -1.7 & -3.4 \\
\hline CFL3D & SX1/UX9 & No & SA-RC (FT) & 2.0434 & 0.3305 & -0.4923 & -0.2 & -0.8 & -2.2 \\
\hline CFL3D & SX1/UX9 & No & $\gamma-R e_{\theta} \mathrm{SST}$ & 2.0581 & 0.3312 & -0.4996 & 0.5 & -0.6 & -0.7 \\
\hline CFL3D & SX1/UX9 & No & $\gamma-R e_{\theta} \mathrm{SST}-\mathrm{RC}$ & 2.0732 & 0.3345 & -0.5072 & 1.3 & 0.4 & 0.8 \\
\hline \multicolumn{10}{|l|}{$\alpha=28^{\circ}$} \\
\hline Experiment & & & & 2.8952 & 0.6776 & -0.4559 & & & \\
\hline FUN3D & UH6 & No & SA (FT) & 2.8478 & 0.6692 & -0.4257 & -1.6 & -1.2 & -6.6 \\
\hline FUN3D & UH16 & No & SA (FT) & 2.8470 & 0.6650 & -0.4250 & -1.7 & -1.9 & -6.8 \\
\hline FUN3D & UH16 & Yes & $\mathrm{SA}(\mathrm{FT})$ & 2.7950 & 0.6537 & -0.4133 & -3.5 & -3.5 & -9.3 \\
\hline FUN3D & UH16 & Yes & SA-RC (FT) & 2.7936 & 0.6581 & -0.4053 & -3.5 & -2.9 & -11.1 \\
\hline FUN3D & UH16 & Yes & $\gamma-R e_{\theta} \mathrm{SST}$ & 2.8674 & 0.6746 & -0.4390 & -1.0 & -0.4 & -3.7 \\
\hline FUN3D & SX1/UX9 & No & SA (FT) & 2.8594 & 0.6650 & -0.4288 & -1.2 & -1.9 & -5.9 \\
\hline CFL3D & SX1/UX9 & No & $\mathrm{SA}(\mathrm{FT})$ & 2.8457 & 0.6613 & -0.4319 & -1.7 & -2.4 & -5.3 \\
\hline CFL3D & SX1/UX9 & No & SST (FT) & 2.8073 & 0.6476 & -0.4186 & -3.0 & -4.4 & -8.2 \\
\hline CFL3D & SX1/UX9 & No & SA-R (FT) & 2.8573 & 0.6632 & -0.4358 & -1.3 & -2.1 & -4.4 \\
\hline CFL3D & SX1/UX9 & No & SA-RC (FT) & 2.8915 & 0.6754 & -0.4472 & -0.1 & -0.3 & -1.9 \\
\hline CFL3D & SX1/UX9 & No & $\gamma-R e_{\theta} \mathrm{SST}$ & 2.9010 & 0.6743 & -0.4519 & 0.2 & -0.5 & -0.9 \\
\hline CFL3D & SX1/UX9 & No & $\gamma-R e_{\theta} \mathrm{SST}-\mathrm{RC}$ & 2.9038 & 0.6756 & -0.4521 & 0.3 & -0.3 & -0.8 \\
\hline
\end{tabular}


In the right-hand column (Figs. 4(b), (d), and (f)), FUN3D results are shown for the UH16 medium grids both without ("no Brkts") and with ("Brkts") brackets. FUN3D's SA results on the configuration without brackets were very similar to those of CFL3D. Although not shown for clarity, the FUN3D force and moment results for the UH6 grid were very similar to the UH16 fully turbulent SA results through stall. Using SA fully turbulent with brackets resulted in $C_{L, \text { max }} \approx 2.82$, around $6 \%$ too low. Use of the 4-equation $\gamma-R e_{\theta}$ SST model resulted in lift levels on the grid with brackets very close to experiment (similar to SA fully turbulent on the grid without brackets). However, at $\alpha=34^{\circ}$, the $\gamma-R e_{\theta}$ SST solution stalled (too early) on the grid with brackets and did not converge.

Thus, when computing on a grid that includes brackets, CFD results tended to more closely approach experimental levels over most of the lift curve when transition was included. As understood from these results (in concert with earlier workshop studies mentioned in the introduction), there are competing mechanisms at play when attempting to predict global forces and moments for the NASA Trapezoidal Wing. In terms of lift: including brackets tends to decrease lift, refining the grid tends to increase it, and including transition tends to increase it. Turbulence model also has a clear effect, with SA tending to produce less separation than SST, all other things being equal. Additional turbulence model influences will be explored below.

\section{B. Surface Pressures}

Figure 5 on page 14 and Fig. 6 on page 15 show surface pressure coefficients at selected stations for $\alpha=13^{\circ}$ and $28^{\circ}$ by using CFL3D and FUN3D, respectively. At the upper surface flap forward station, there was a trend of lower pressures (more negative $C_{p}$ ) for the $\gamma-R e_{\theta}$ SST model, followed by the SA model, and higher pressures for the SST model. Generally, the results from the $\gamma-R e_{\theta}$ SST model agreed best with experiment along this station, and fully turbulent SST the worst. The FUN3D results on the grid with brackets captured the "scalloping" effect of the brackets on the pressures, in better agreement with experiment. All models performed poorly near the wing tip region, outboard of $y \approx-80$ inches. This was also the case at the first workshop, and was most likely due to inadequate resolution of the wing tip vortex. Either insufficient grid density or deficiencies in turbulence modeling (or a combination of both) was probably to blame. A subsequent study by Lee and Pulliam ${ }^{18}$ demonstrated the important influence of grid density in the wing tip region.

At the 50\% span station on the flap, the $\gamma-R e_{\theta}$ SST model yielded results closer to experiment than fully turbulent SA or SST at $\alpha=13^{\circ}$. The tendency of this transition model to yield lower pressures over the flap upper surface can clearly be seen. At $\alpha=28^{\circ}$, none of the models agreed well with experiment. However, the FUN3D results were somewhat closer to experiment than those of CFL3D, probably because of the bracket influence at this particular station. ${ }^{1}$

Finally, at the $85 \%$ span station on the flap, similar trends were seen for the three turbulence models. However, in some cases the $\gamma-R e_{\theta}$ SST model agreed best with experiment, while in others the fully turbulent SA model was closer. The brackets also had an influence at this span station: including them improved the comparison with lower surface flap pressures at both $\alpha=13^{\circ}$ and $28^{\circ}$.

\section{Off-Body Velocity Profiles at $\alpha=28^{\circ}$}

Recently, off-body velocity profiles (measured using 7-hole probes) at a limited set of locations in the NASA Langley 14- by 22 -foot wind tunnel were made available in Hannon et al. ${ }^{4}$ One set of these experimental data were taken at $\alpha=28^{\circ}$. Velocity magnitude results on the SX1/UX9 grid are shown in Fig. 7 on page 16, using SA (fully turbulent), SST (fully turbulent), and $\gamma-\operatorname{Re}_{\theta} \mathrm{SST}$ at four locations normal to the wing and flap upper surfaces. These surface locations are near: $x=17.5, y=-13.6 ; x=64.2, y=-71.5 ; x=69.7, y=-71.9 ; x=75.6, y=-72.4$ inches. Unfortunately, the experimental data probe did not traverse close enough to the surface over the main element (Figs. 7(a) and (b)), so it missed the boundary layer and most of the slat wake. CFL3D and FUN3D were both run with the SA model (fully turbulent) on this same grid, and their results were very similar at each station. CFD results at the aft flap station (in the region where surface streamlines indicated separated flow) were all poor, but at the other three stations the CFD results agreed fairly well with experiment, and the $\gamma-R e_{\theta}$ SST model performed generally best. It yielded particularly good results for the wakes at the forward flap station. Experimental data were also available for the respective $x, y$, and $z$ components of velocity at three of the stations, but plotting these did not reveal any additional information helpful for turbulence model validation.

Figure 8 on page 17 compares several FUN3D results on the UH16 grid. Fully turbulent SA model results, on grids both with and without brackets, were very similar to each other. The $\gamma-R e_{\theta}$ SST model results were generally better compared to experiment. However, all results on the UH16 grid were much worse compared to experiment (wakes were more diffused) than results on the SX1/UX9 grid (see Fig. 7). By inspecting cuts of the respective grids near 
the outboard span stations (Fig. 9 on page 18), it is clear that the SX1/UX9 grid provided much greater wall-normal grid density than UH16 in the regions above the wing where the wakes from upstream elements pass. This greater grid density allowed more accurate wake resolution. Providing grids with pre-specified wake clustering is currently a challenge for many unstructured grid generation codes.

Experimental off-body velocity and turbulence profile measurements are extremely useful for CFD validation studies such as this, because they can highlight specific deficiencies in grid resolution and turbulence modeling. As seen in the current velocity results, inadequate grid resolution in wake regions can produce diffuse wake structures that do not resemble the true physics. It is unclear what impact the diffused wakes have on global quantities of interest, because so many other factors also come into play. However, Smith ${ }^{38}$ made the observation that when wakes and boundary layers merge too quickly, early separation will set in. This implies that under-resolution of the wakes in a CFD computation could influence stall prediction.

\section{Transition Effects}

Although the $\gamma-\operatorname{Re}_{\theta}$ SST model yielded very good results for this configuration, the model did not converge well as implemented in both CFL3D and FUN3D. In particular, the residual of the $\gamma$ turbulence equation tended to stall, typically not decreasing beyond a certain level (depending on the case). An example using CFL3D at $\alpha=13^{\circ}$ is shown in Fig. 10 on page 18. Here, the mean flow density residual (red line) was converging, and lift coefficient (blue line) was settling to nearly a constant level. However, the residual for the $\gamma$ turbulence equation (green line) was oscillatory near $10^{-7}$, and not dropping. This represents residual convergence of only about one order of magnitude from this equation's initial (maximum) level. Nonetheless, in general we have observed that the patterns of laminar and turbulent flow generally "converge" in the sense that they achieve a fixed pattern then do not change noticeably. Naturally, poor convergence of turbulence equation residuals is a source of concern and potential uncertainty because an adequately converged mean flow final result is not assured. More work needs to be done to investigate the numerical behavior of the $\gamma-R e_{\theta}$ SST model.

The difference between the flowfields in the flap region using SST and the $\gamma-\operatorname{Re}_{\theta}$ SST models is exemplified in Fig. 11 on page 19. The fully turbulent SST model yielded lower velocities over the crest of the flap than the $\gamma-R_{\theta}$ SST model (Figs. 11(a) and (b) at a representative span station), and SST produced a larger region of separated flow on the aft half of the flap. The nondimensional eddy viscosity levels for the two models are shown in close-ups over the front part of the flap at the same representative span station in Figs. 11(c) and (d). Generally, levels of $\mu_{t} / \mu_{\text {ref }}>1$ are considered turbulent. The $\gamma-R_{\theta}$ SST model produced "laminar" levels of eddy viscosity (seen as thin dark blue regions) over about the first $40 \%$ of the flap upper surface, as well as over the entire lower surface at this $y$-station. According to Smith, ${ }^{38}$ extensive laminar flow helps to delay separation because it reduces $R e_{\theta}$. Further, thin boundary layers can withstand stronger adverse gradients than thick ones. Upstream transition effect on downstream wake shape may also play a role. ${ }^{23,39}$ The upper surface streamlines for the two models are shown in Figs. 11(e) and (f). The $\gamma-R e_{\theta}$ SST model yielded significantly less separated flow on the flap than fully turbulent SST.

Next the predicted transition locations from the $\gamma-R e_{\theta}$ SST model are compared with those of Eliasson et al. ${ }^{17}$ To visualize the laminar and turbulent regions on the Trapezoidal Wing, an approximated turbulence index $i_{t}$ was used, based on the one described in Spalart and Allmaras. ${ }^{19}$ This index was designed to yield a value close to zero in a laminar region and close to one in a turbulent region for the SA model. Although not designed with other turbulence models in mind (which may have different asymptotic behavior near the wall), translating any model's eddy viscosity to an equivalent $\tilde{\nu}$ and making use of the Spalart-Allmaras formula has been found to be valuable for visualizing regions where there are clear demarcations between laminar and turbulent flow.

Figure 12 on page 20 shows $i_{t}$ contours compared with locations predicted by Eliasson et al. using the $e^{N}$ method, with $N=10$. Eliasson et al. found this latter method (in combination with SA) to yield reasonably good correlations with the experimental data of McGinley et al. ${ }^{22}$ as well as produce good overall force and moment results in computations compared to experiment. On the upper surfaces (Figs. 12(a), (c), and (e)), there was generally good correlation between the methods. On the lower surfaces (Figs. 12(b), (d), and (f)), the correlation between the methods was fairly good on the flap (where mostly laminar flow was indicated), but was not as good on the main element. Here, the $\gamma-R_{\theta}$ SST model indicated either transitional or turbulent values in very unusual patterns, whereas the $e^{N}$ method indicated more significant regions of laminar flow. In contrast, McGinley et al. noted primarily turbulent flow near the mid-chord region of the main element lower surface in the experiment. As mentioned above, the current approximate turbulence index is only helpful for determining regions that are very definitively laminar or turbulent. On the lower surface of the main element the $\gamma-R e_{\theta}$ SST model produced results that are indeterminate with this metric. Although not shown, $i_{t}$ contours for the $\gamma-R e_{\theta}$ SST model in FUN3D on the UH16 grid with brackets looked similar to those in Fig. 12, although the presence of the brackets caused some non-uniformity in the index values on the adjacent downstream 
element.

In Fig. 13 on page 21, examples of the combined effects of brackets and transition on $C_{L}$ at $\alpha=13^{\circ}$ are shown. With no brackets and SA (fully turbulent), the lift was computed about $2.6 \%$ too low. Adding brackets lowered lift (making it 3.3\% low). However, including transition on the grid with brackets-either through fixing transition a priori or using the $\gamma-R e_{\theta}$ SST model—brought the lift back up (making it $1.7 \%$ low, near the lower experimental uncertainty bounds).

\section{E. Rotation and Curvature Correction Effects}

Looking beyond the trends caused by including transition effects, we now examine the effects of including various rotation and curvature corrections in the turbulence models. These corrections are expected to better account for areas of the flow where either pure rotation should not produce additional turbulence, or where streamline curvature near solid walls should influence turbulence (reduction near convex curvature, enhancement near concave curvature). Figure 14 on page 21 shows nondimensional vorticity contours for $\alpha=13^{\circ}$, for two representative cases without and with brackets. These plots show flowfield features for which rotation and curvature corrections may be important. In particular, the wing tip sheds a strong vortex that convects downstream. When brackets are included, there are other smaller vortical features shed inboard as well. Including rotation and curvature corrections had a significant influence on the strength of the shed vortices. For example, at the aft-most station shown ( $x=90$ inches), peak tip vortex strength was increased (compared to baseline SA or SST, which were similar) by $15 \%$ with SA-R and by $21 \%$ with SA-RC. The $\gamma-R e_{\theta}$ SST model increased tip vortex strength by $10 \%$, and $\gamma-R e_{\theta}$ SST-RC increased tip vortex strength by $28 \%$ over the baseline models.

Figure 15 on page 22 summarizes the effects of the various rotation/curvature corrections. In Figs. 15(a) and (b), lift values at the two angles of attack of $\alpha=13^{\circ}$ and $28^{\circ}$ are shown from CFL3D with different turbulence model variants. Use of SA-R, SA-RC, or SST-RC had the influence of increasing $C_{L}$ (trends were similar for FUN3D). The SA-RC model had a larger influence than SA-R. The SST-RC correction was run in conjunction with the transition model ( $\gamma-R e_{\theta}$ SST-RC), and yielded higher lift than $\gamma-R e_{\theta}$ SST alone. Although not shown in figures, effects on drag and moment can be seen in Table 2. Including transition and rotation/curvature corrections both had the tendency to increase drag and decrease moment, usually improving correlation with experiment.

Finally, we address the question of whether the turbulence model rotation and curvature corrections improved surface pressure predictions in the problematic wing tip region. Figures 15(c) and (d) show spanwise surface pressure coefficient comparisons at the flap forward span station. Two angles of attack $\left(\alpha=13^{\circ}\right.$ and $\left.28^{\circ}\right)$ are shown for CFL3D on the SX1 grid. Generally, the rotation and curvature corrections tended to decrease the upper surface flap pressures, but none of the models improved the tip region predictions. As implied in Lee and Pulliam, ${ }^{18}$ significantly greater grid resolution is probably required in the wing tip region. Only with adequate grid resolution will it be possible to conduct meaningful studies of the influence of turbulence models on the flow physics in this area of the flow. Figure 15(e) shows results for FUN3D on the UH16 grid with brackets. Here, for clarity only SA and SA-RC results are shown (both with transition set a priori). Again, the rotation/curvature correction yielded lower upper surface pressures along most of this station on the flap, in better agreement with experiment out to approximately $y=-65$ inches, but did not improve the predictions near the wing tip. In Fig. 15(f) the differences in predicted $C_{p}$ between SA-RC and SA are shown in a contour plot. Most of the largest differences occurred near the wing tip. On the flap, the $C_{p}$ levels from SA-RC were mostly lower over the front part and mostly higher near the trailing edge.

\section{Conclusions}

The first High Lift Prediction Workshop, which used the NASA Trapezoidal Wing experimental data, brought several CFD issues to light. Among these were the important influences of grid resolution, brackets, and transition. The current paper's goal was to make use of many of the lessons learned from the workshop and subsequent independent studies, and attempt to further advance our understanding of how to improve RANS modeling of this type of flowfield.

Most importantly, transition influence was included. Two methods were tested: (1) manually zeroing out turbulence production in pre-determined regions, and (2) use of a recently published 4-equation transition model. The former method proved to be tedious in general, and impractical to use at high angles of attack. The latter method proved to be a very effective engineering tool for this complex three-dimensional high-lift flow, in spite of some convergence difficulties associated with the model. The 4-equation transition model predicted laminar regions fairly well in comparison with an $e^{N}$ method, and tended to increase lift and decrease moment, in better agreement with experiment than fully turbulent computations. Upper surface flap separation was reduced, and agreement with experimental 
surface pressures and velocity profiles was improved.

The current study also confirmed that including the effects of transition in combination with the brackets in the CFD model improved agreement with the experimental data at pre-stall angles of attack. However, the unstructured grids with brackets that were used had poor resolution in the wake regions above the main and flap elements. This poor local grid resolution yielded diffused velocity profile predictions. The precise effects of these diffuse predictions are not known, but it is possible that they could influence the ability of the computed flowfield to resist stall. ${ }^{38}$ Recentlyreleased experimental velocity profiles were valuable for demonstrating the influence of grid and turbulence model on wake shape predictions. Several different turbulence model enhancements to account for rotation and curvature were tested. These all generally had the desirable effects of increasing lift (reducing upper surface pressures) and improving the resolution of the wing tip vortex as it convected downstream. However, none of the models improved the predictions of the wing surface pressures near the wing tip, where the grid resolution was likely inadequate.

Consistent and reliable prediction of the flowfield near stall angle remains elusive. There are offsetting factors (e.g., increasing grid resolution and accounting for transition tending to increase lift; accounting for brackets tending to decrease it), so that it is possible to get a reasonably good $C_{L, \max }$ prediction for the wrong reasons. As a result, firm conclusions about the efficacy of specific turbulence models near $C_{L, \max }$ are difficult to make without addressing all of the influencing factors such as transition, geometry, and grid resolution simultaneously. Accurate predictions will require transition modeling and inclusion of all important geometric features such as brackets, as well as adequate grid resolution. Particular attention needs to be paid to the wing tip and wake regions of the grid, either through a priori grid refinement or by using grid adaption.

\section{References}

\footnotetext{
${ }^{1}$ Rumsey, C. L., Slotnick, J. P., Long, M., Stuever, R. A., and Wayman, T. R., "Summary of the First AIAA CFD High-Lift Prediction Workshop," Journal of Aircraft, Vol. 48, No. 6, 2011, pp. 2068-2079.

${ }^{2}$ Slotnick, J. P., Hannon, J. A., and Chaffin, M., "Overview of the First AIAA CFD High Lift Prediction Workshop (Invited)," AIAA Paper 2011-862, January 2011.

${ }^{3}$ Johnson, P. L., Jones, K. M., and Madson, M. D., "Experimental Investigation of a Simplified 3D High Lift Configuration in Support of CFD Validation," AIAA Paper 2000-4217, August 2000.

${ }^{4}$ Hannon, J. A., Washburn, A. E., Jenkins, L. N., and Watson, R. D., "Trapezoidal Wing Experimental Repeatability and Velocity Profiles in the 14- by 22-Foot Subsonic Tunnel (Invited),” AIAA Paper 2012-0706, January 2012.

${ }^{5}$ Long, M. and Mavriplis, D., "NSU3D Results for the First AIAA High Lift Prediction Workshop,” AIAA Paper 2011-863, January 2011.

${ }^{6}$ Steed, R. G. F., "High Lift CFD Simulations with an SST-Based Predictive Laminar to Turbulent Transition Model," AIAA Paper 2011-864, January 2011.

${ }^{7}$ Wiart, L. and Meunier, M., "Computational Assessment of the HiLiftPW-1 Trap-Wing Model Using the elsA CFD Software," AIAA Paper 2011-865, January 2011.

${ }^{8}$ Sclafani, A. J., Slotnick, J. P., Vassberg, J. C., Pulliam, T. H., and Lee, H. C., "OVERFLOW Analysis of the NASA Trap Wing Model from the First High Lift Prediction Workshop," AIAA Paper 2011-866, January 2011.

${ }^{9}$ Eliasson, P., Peng, S.-H., and Hanifi, A., "Improving the Prediction for the NASA High-Lift Trap Wing Model," AIAA Paper 2011-867, January 2011.

${ }^{10}$ Reyes, D. A., Girimaji, S. S., Pandya, M., and Abdol-Hamid, K. S., "Computations of High-Lift Wing-Body Configuration on Unstructured Grids using $k$ - $\omega$ Models," AIAA Paper 2011-868, January 2011.

${ }^{11}$ Fares, E. and Nolting, S., "Unsteady Flow Simulation of a High-Lift configuration using a Lattice Boltzmann Approach," AIAA Paper 2011-869, January 2011.

${ }^{12}$ Park, M. A., Lee-Rausch, E. M., and Rumsey, C. L., "FUN3D and CFL3D Computations for the First High Lift Prediction Workshop," AIAA Paper 2011-936, January 2011.

${ }^{13}$ Murayama, M., Yamamoto, K., and Tanaka, K., "CFD Comparison Study for Trapezoidal High-Lift Wing Configurations by Structured and Unstructured Mesh Method," AIAA Paper 2011-937, January 2011.

${ }^{14}$ Crippa, S., Melber-Wilkending, S., and Rudnick, R., "DLR Contribution to the First High Lift Prediction Workshop,” AIAA Paper 2011-938, January 2011.

${ }^{15}$ Antunes, A. P., Azevedo, J. L. F., and da Silva, R. G., "Numerical Simulations of Turbulent Flow over a High-Lift Configuration," AIAA Paper 2011-3006, June 2011.

${ }^{16}$ Pandya, M. J., Abdol-Hamid, K. S., and Parlette, E. B., "CFD Computations for a Generic High-Lift Configuration Using TetrUSS," AIAA Paper 2011-3008, June 2011.

${ }^{17}$ Eliasson, P., Hanifi, A., and Peng, S.-H., "Influence of Transition on High-Lift Prediction for the NASA Trap Wing Model," AIAA Paper 2011-3009, June 2011.

${ }^{18}$ Lee, H. C. and Pulliam, T. H., "Effect of Using Near and Off-body Grids with Grid Adaption to Simulate Airplane Geometries," AIAA Paper 2011-3985, June 2011.

${ }^{19}$ Spalart, P. R. and Allmaras, S. R., “A One-Equation Turbulence Model for Aerodynamic Flows,” Recherche Aerospatiale, No. 1, 1994, pp. $5-21$.

${ }^{20}$ Menter, F. R., “Two-Equation Eddy-Viscosity Turbulence Models for Engineering Applications,” AIAA Journal, Vol. 32, No. 8, 1994, pp. $1598-1605$.
} 
${ }^{21}$ Langtry, R. B. and Menter, F. R., "Correlation-Based Transition Modeling for Unstructured Parallelized Computational Fluid Dynamics Codes," AIAA Journal, Vol. 47, No. 12, 2009, pp. 2894-2906.

${ }^{22}$ McGinley, C. B., Jenkins, L. N., Watson, R. D., and Bertelrud, A., "3-D High-Lift Flow-Physics Experiment - Transition Measurements," AIAA Paper 2005-5148, June 2005.

${ }^{23}$ Rumsey, C. L., Gatski, T. B., Ying, S. X., and Bertelrud, A., "Prediction of High-Lift Flows Using Turbulent Closure Models," AIAA Journal, Vol. 36, No. 5, 1998, pp. 765-774.

${ }^{24}$ Dacles-Mariani, J., Zilliac, G. G., Chow, J. S., and Bradshaw, P., "Numerical/Experimental Study of a Wingtip Vortex in the Near Field," AIAA Journal, Vol. 33, No. 9, 1995, pp. 1561-1568.

${ }^{25}$ Shur, M. L., Strelets, M. K., Travin, A., and Spalart, P. R., "Turbulence Modeling in Rotating and Curved Channels: Assessing the SpalartShur Correction," AIAA Journal, Vol. 38, No. 5, 2000, pp. 784-792.

${ }^{26}$ Hellsten, A., “Some Improvements in Menter's $k-\omega$ SST Turbulence Model,” AIAA Paper 98-2554, June 1998.

${ }^{27}$ Mani, M., Ladd, J. A., and Bower, W. W., "Rotation and Curvature Correction Assessment for One- and Two-Equation Turbulence Models," Journal of Aircraft, Vol. 41, No. 2, 2004, pp. 268-273.

${ }^{28}$ Krist S. L., Biedron R. T., and Rumsey C. L., “CFL3D User’s Manual (Version 5.0),” NASA/TM-1998-208444, June 1998.

${ }^{29}$ Anderson, W. K. and Bonhaus, D. L., "An Implicit Upwind Algorithm for Computing Turbulent Flows on Unstructured Grids," Computers and Fluids, Vol. 23, No. 1, 1994, pp. 1-22.

${ }^{30}$ Menter, F. R., "Improved Two-Equation k-omega Turbulence Models for Aerodynamic Flows," NASA TM 103975, October 1992.

${ }^{31}$ Langtry, R. B., “A Correlation-Based Transition Model using Local Variables for Unstructured Parallelized CFD codes,” PhD Thesis, Institut fur Thermische Stromungsmaschinen und Maschinenlaboratorium, Universitat Stuttgart, 2006.

${ }^{32}$ Kato, M. and Launder, B. E. "The Modeling of Turbulent Flow Around Stationary and Vibrating Square Cylinders," Proc. 9th Symposium on Turbulent Shear Flows, Kyoto, August 1993, pp. 10.4.1-10.4.6.

${ }^{33}$ Durbin, P. A., "On the k-epsilon Stagnation Point Anomaly," International Journal of Heat and Fluid Flow, Vol. 17, 1996, pp. 89-90.

${ }^{34}$ Rumsey, C. L., Gatski, T. B., Anderson, W. K., and Nielsen, E. J., "Isolating Curvature Effects in Computing Wall-Bounded Turbulent Flows," International Journal of Heat and Fluid Flow, Vol. 22, 2001, pp. 573-582.

${ }^{35}$ Jespersen, D. C., Pulliam, T. H., and Buning, P. G., "Recent Enhancements to OVERFLOW," AIAA Paper 97-0644, Reno, NV, 1997.

${ }^{36}$ Neuhart, D. H. and McGinley, C. B., "Free-Stream Turbulence Intensity in the Langley 14- by 22-Foot Subsonic Tunnel," NASA TP-2004213247, August 2004.

${ }^{37}$ Spalart, P. R. and Rumsey, C. L., "Effective Inflow Conditions for Turbulence Models in Aerodynamic Calculations," AIAA Journal, Vol. 45, No. 10, 2007, pp. 2544-2553.

${ }^{38}$ Smith, A. M. O., "High-Lift Aerodynamics," Journal of Aircraft, Vol. 12, No. 6, 1975, pp. 501-530.

${ }^{39}$ Ying, S. X., Spaid, F. W., McGinley, C. B., and Rumsey, C. L., "Investigation of Confluent Boundary Layers in High-Lift Flows," Journal of Aircraft, Vol. 36, No. 3, 1999, pp. 550-562. 


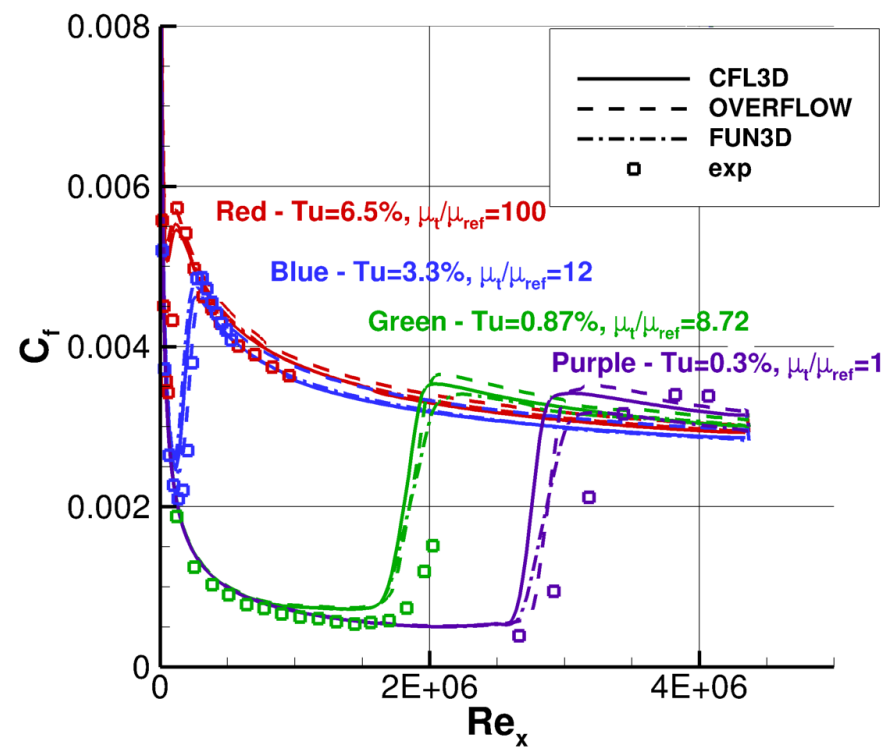

Figure 1: Flat plate skin friction coefficient results using the $\gamma-\operatorname{Re}_{\theta} \operatorname{SST}$ model in three different codes, $M=0.2$.

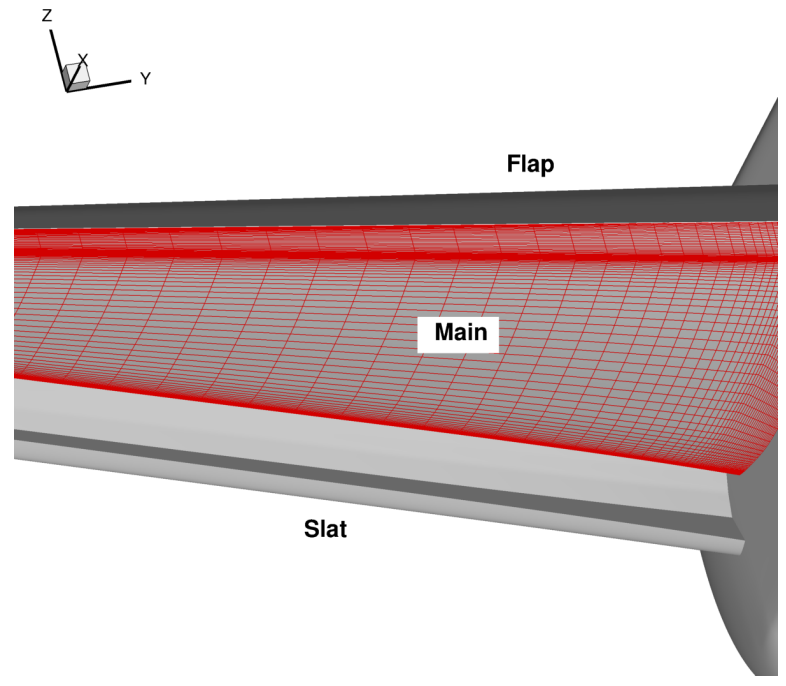

(a) Structured SX1 grid (no brackets).
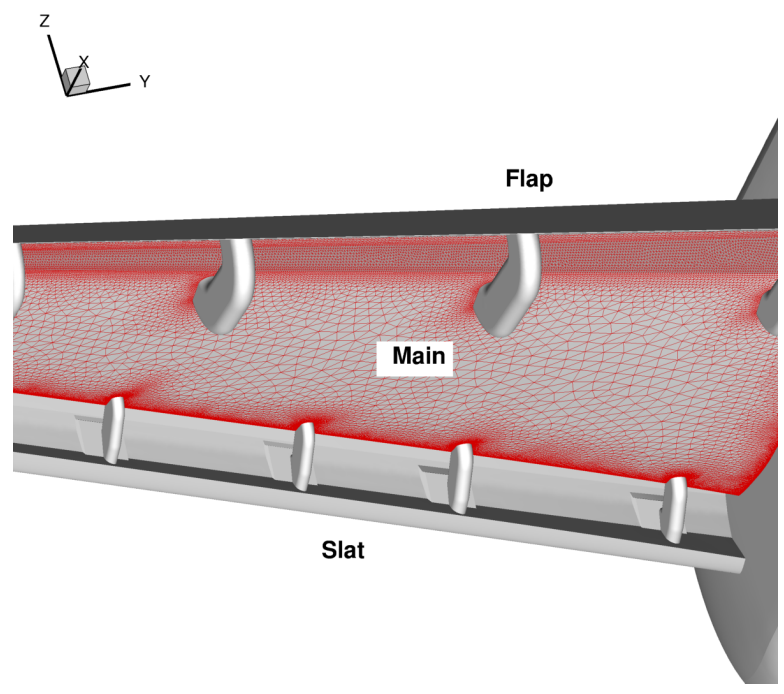

(b) Unstructured UH16 grid (includes brackets).

Figure 2: View of underside of NASA Trapezoidal Wing (looking forward), without and with brackets, and showing surface grid spacing on the main element. 


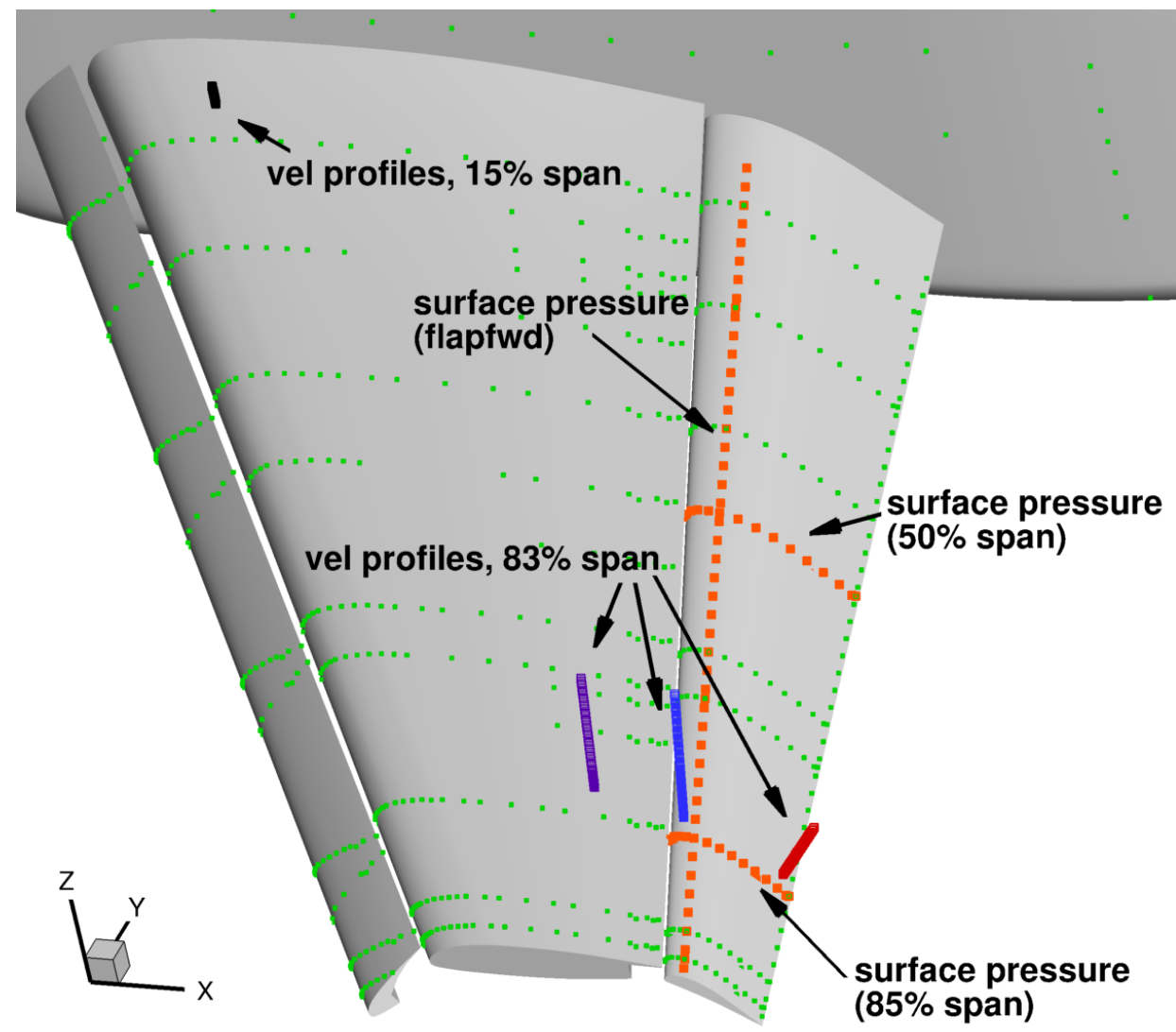

Figure 3: View of pressure tap locations (on surface) and 7-hole-probe locations (normal to surface) over the upper surface of the NASA Trapezoidal Wing (pressure tap locations compared in this study are highlighted in orange). 


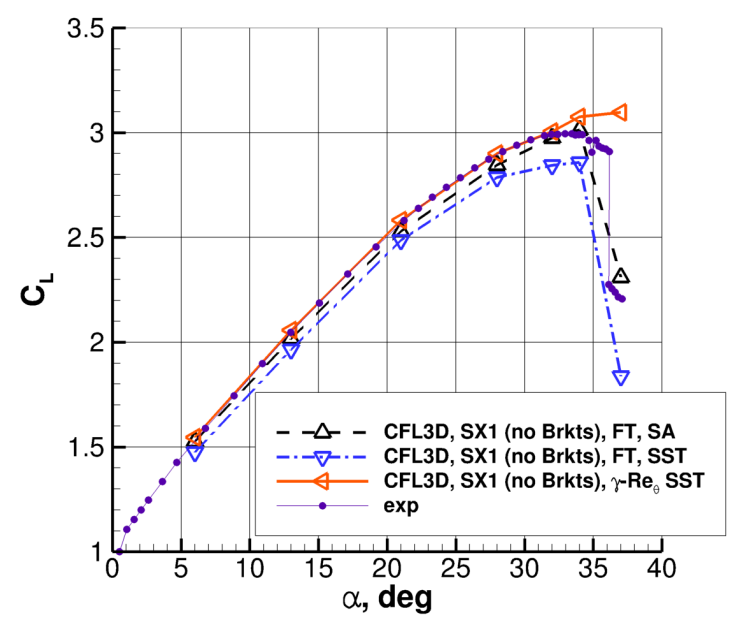

(a) Lift curve, CFL3D.

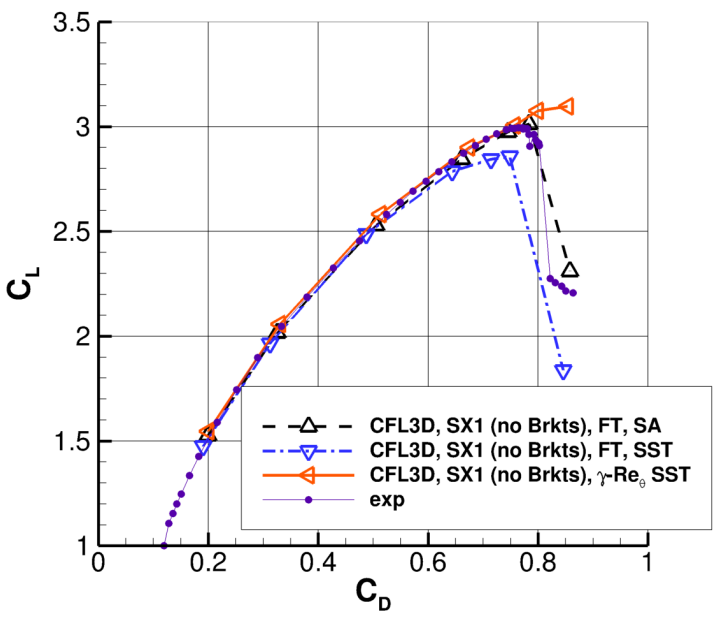

(c) Drag curve, CFL3D.

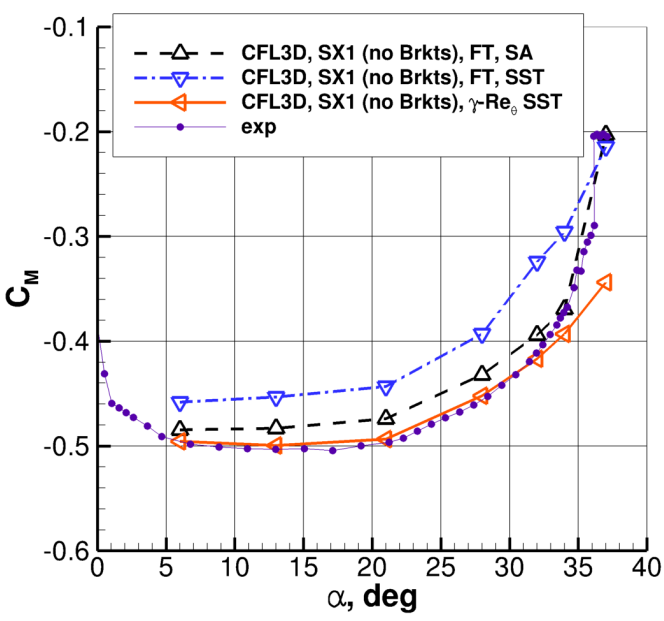

(e) Pitching moment curve, CFL3D.

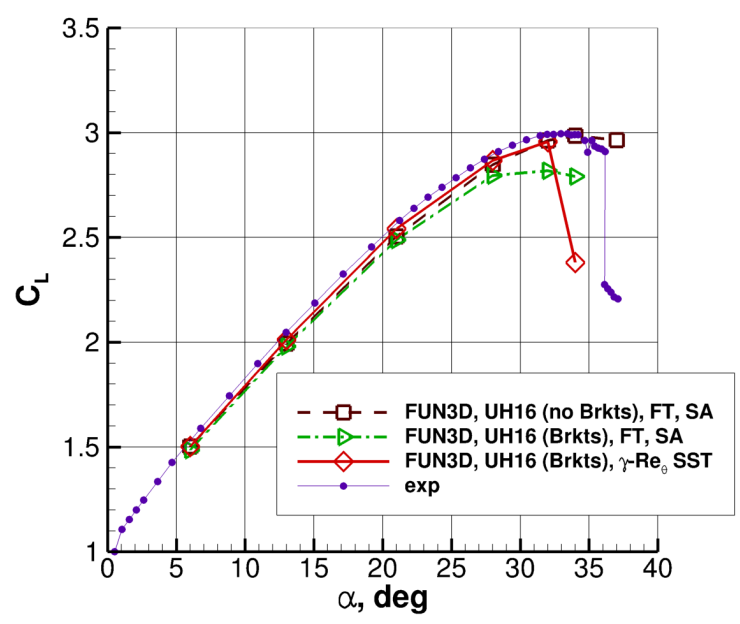

(b) Lift curve, FUN3D.

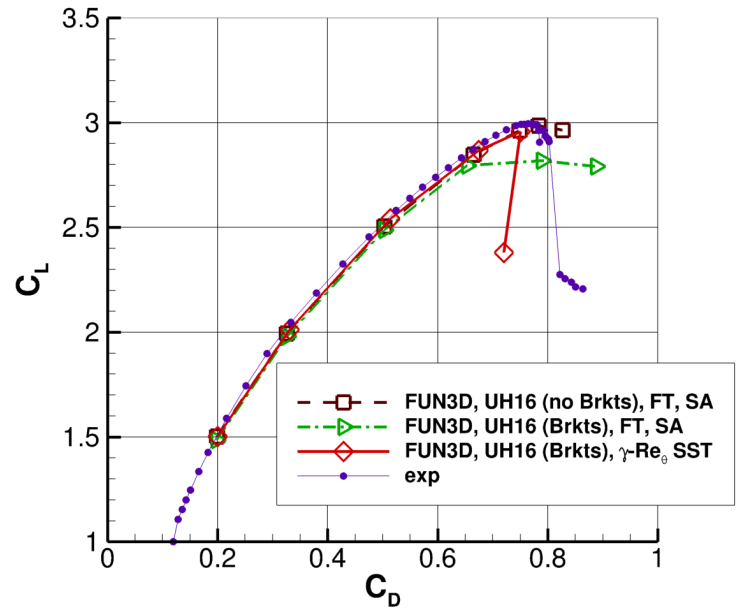

(d) Drag curve, FUN3D.

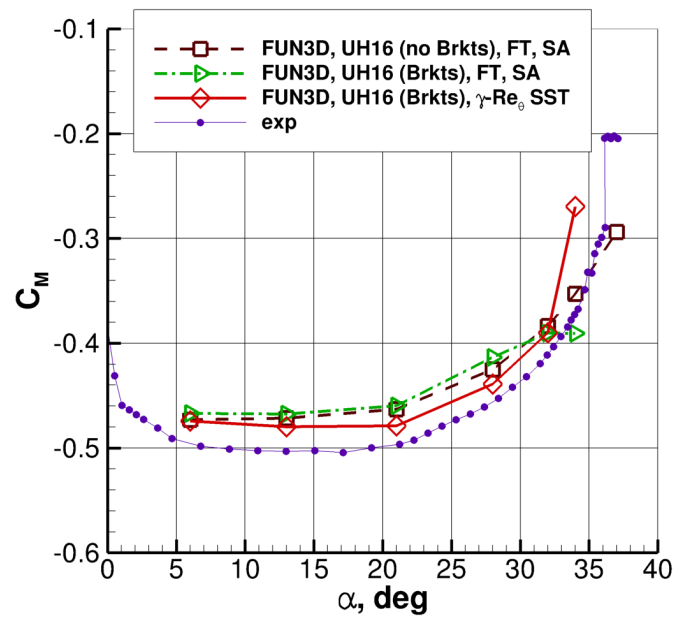

(f) Pitching moment curve, FUN3D.

Figure 4: Force and moment comparisons for NASA Trapezoidal Wing. 


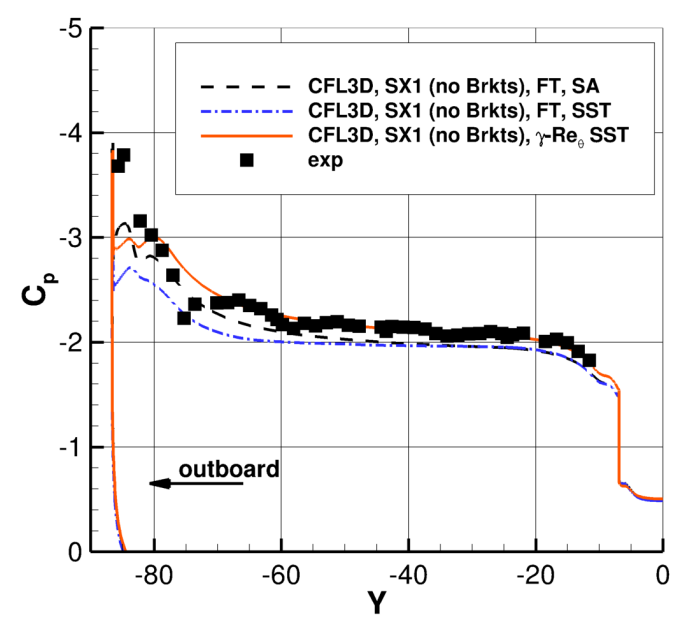

(a) $\alpha=13^{\circ}$, flap forward station.

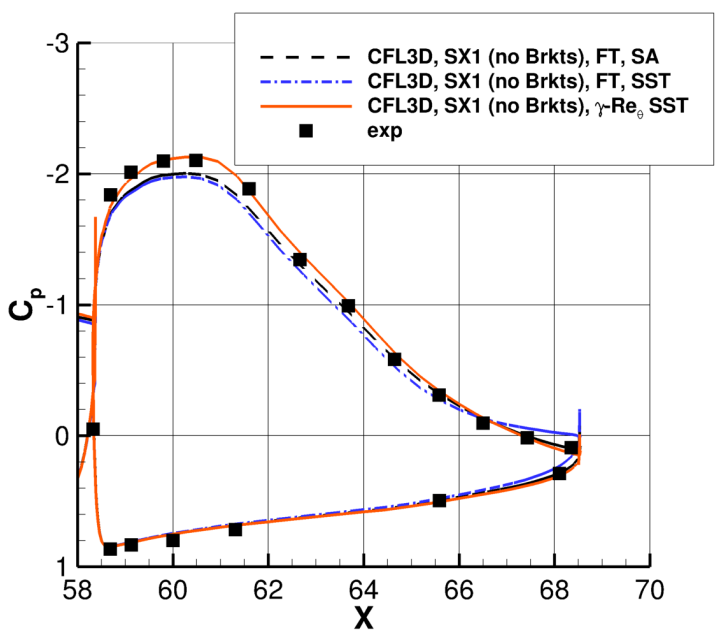

(c) $\alpha=13^{\circ}$, flap at $50 \%$ span station.

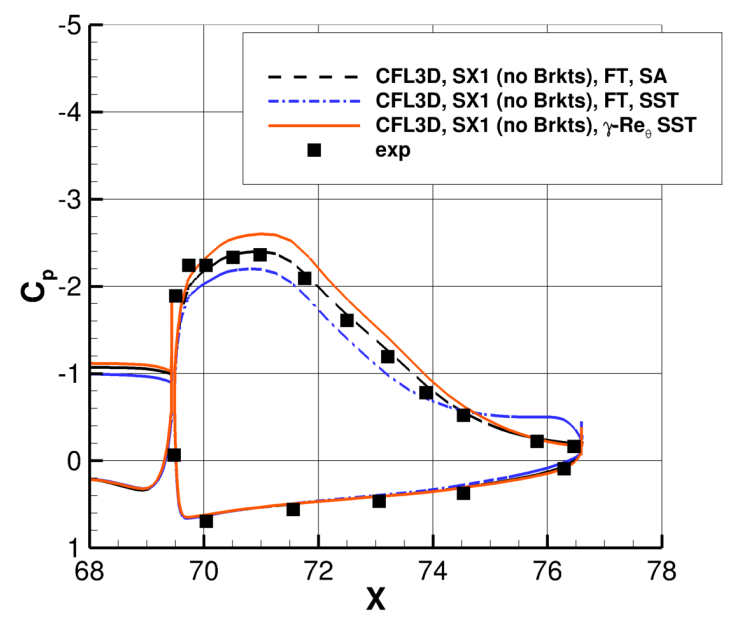

(e) $\alpha=13^{\circ}$, flap at $85 \%$ span station.

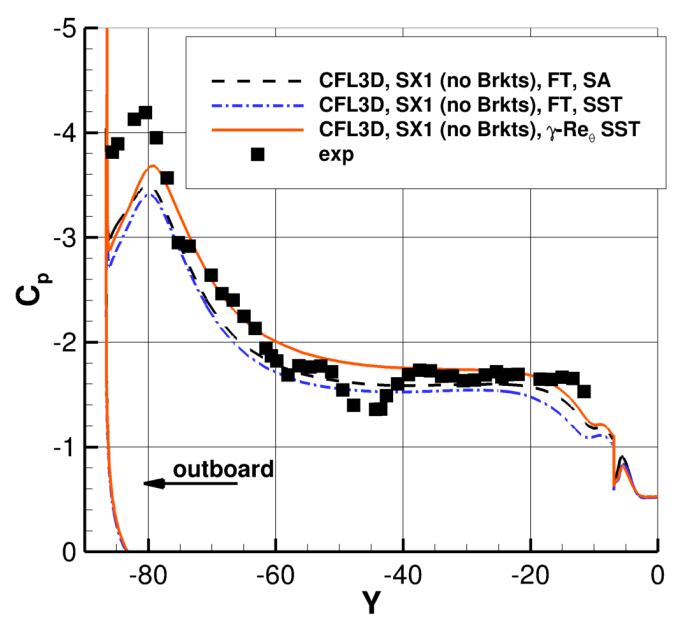

(b) $\alpha=28^{\circ}$, flap forward station.

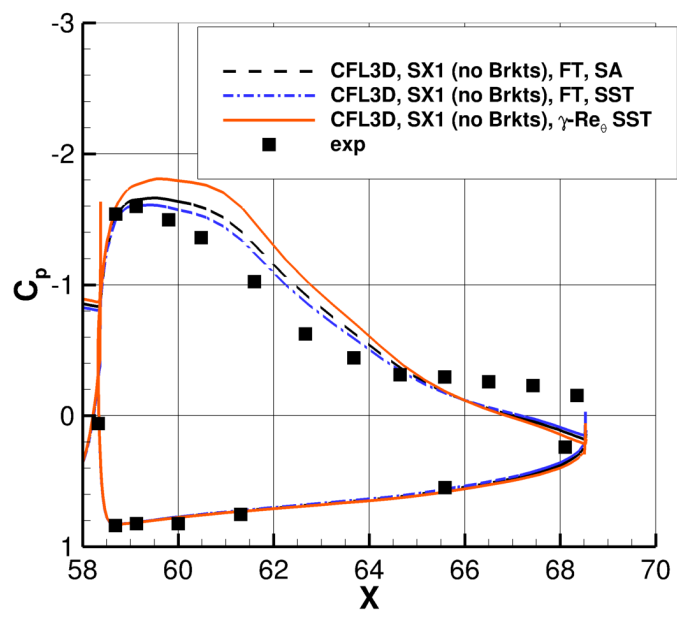

(d) $\alpha=28^{\circ}$, flap at $50 \%$ span station.

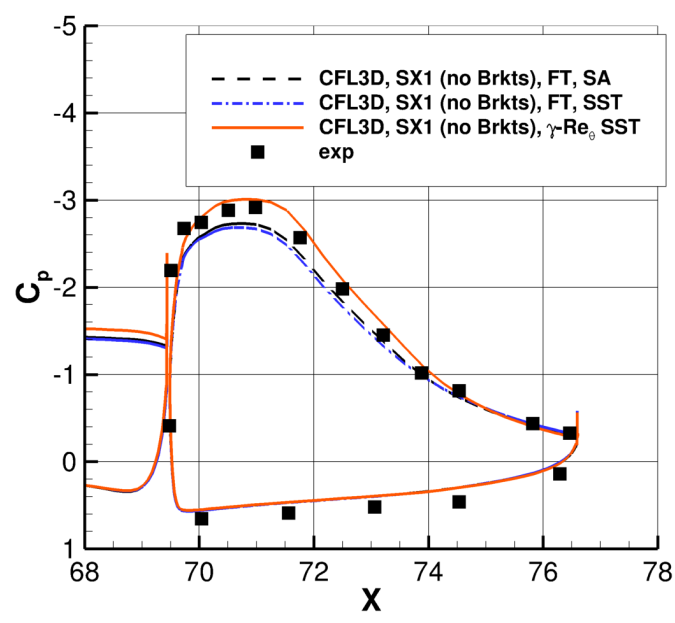

(f) $\alpha=28^{\circ}$, flap at $85 \%$ span station.

Figure 5: Comparison of surface pressure coefficients using CFL3D on SX1 grid. 


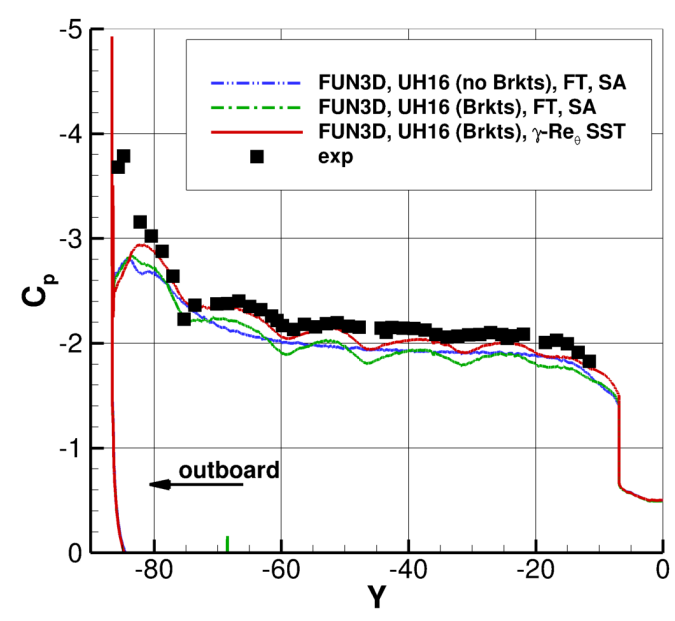

(a) $\alpha=13^{\circ}$, flap forward station.

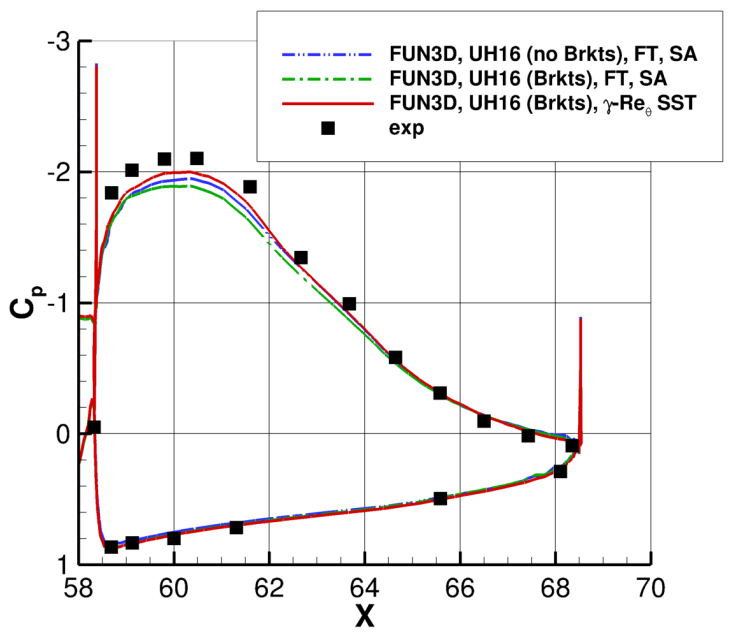

(c) $\alpha=13^{\circ}$, flap at $50 \%$ span station.

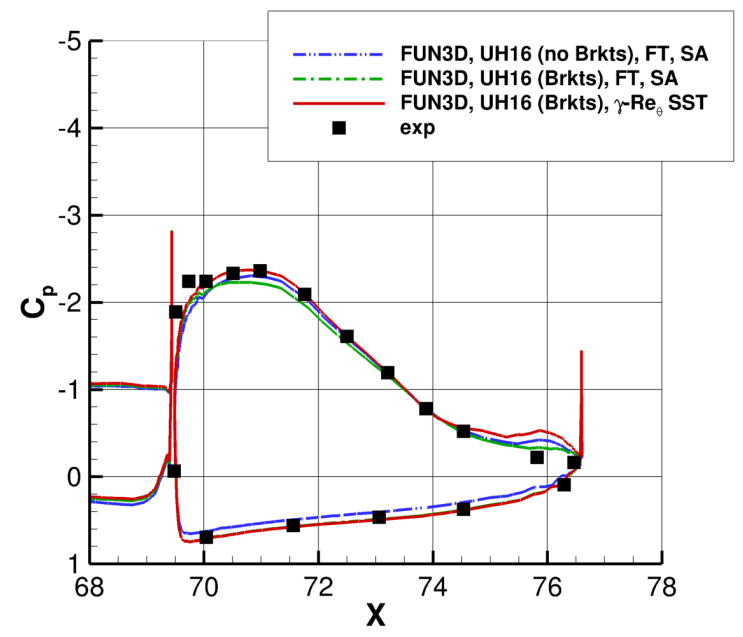

(e) $\alpha=13^{\circ}$, flap at $85 \%$ span station.

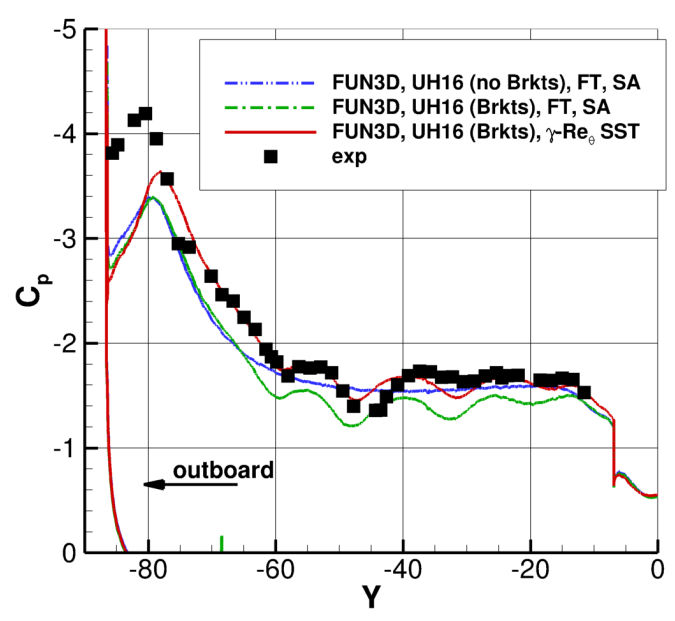

(b) $\alpha=28^{\circ}$, flap forward station.

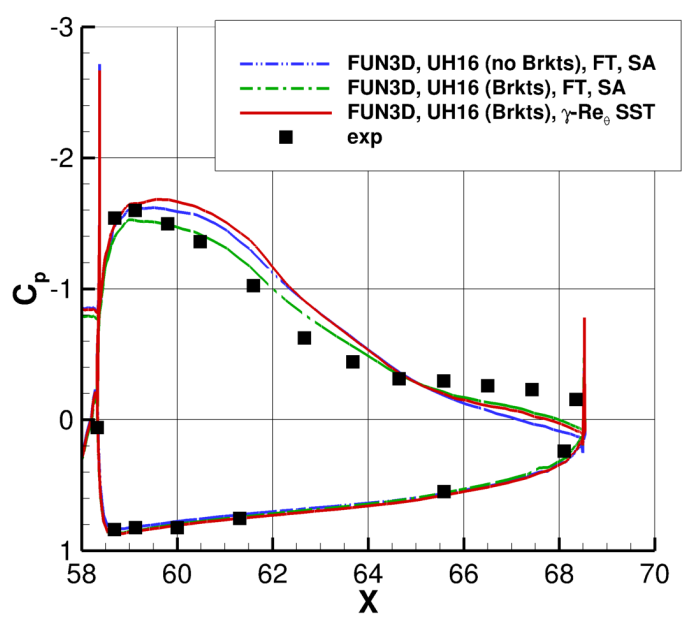

(d) $\alpha=28^{\circ}$, flap at $50 \%$ span station.

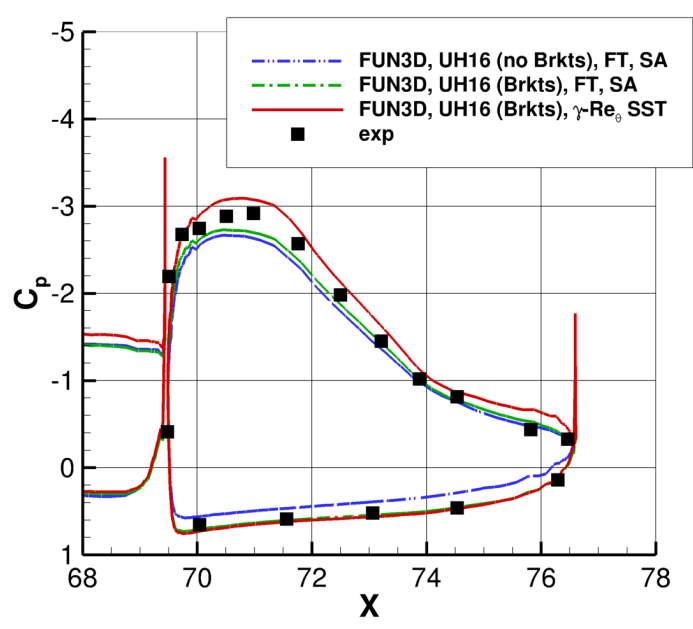

(f) $\alpha=28^{\circ}$, flap at $85 \%$ span station.

Figure 6: Comparison of surface pressure coefficients using FUN3D on UH16 grid. 


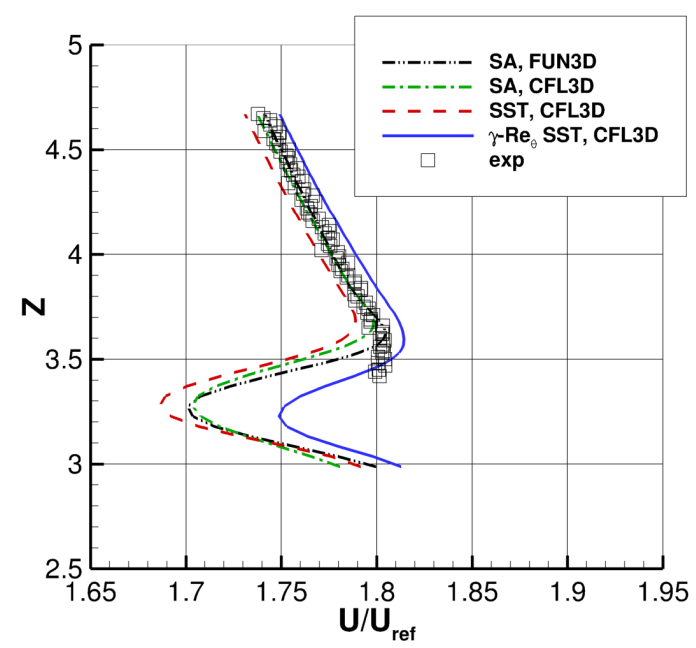

(a) Main element, 15\% span location, near $x=17.5$ inches.

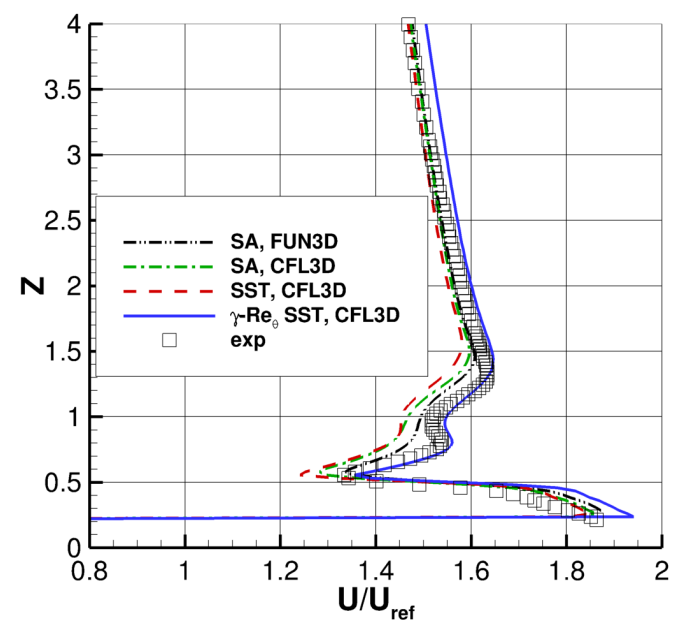

(c) Forward location on flap element, 83\% span location, near $x=69.7$ inches.

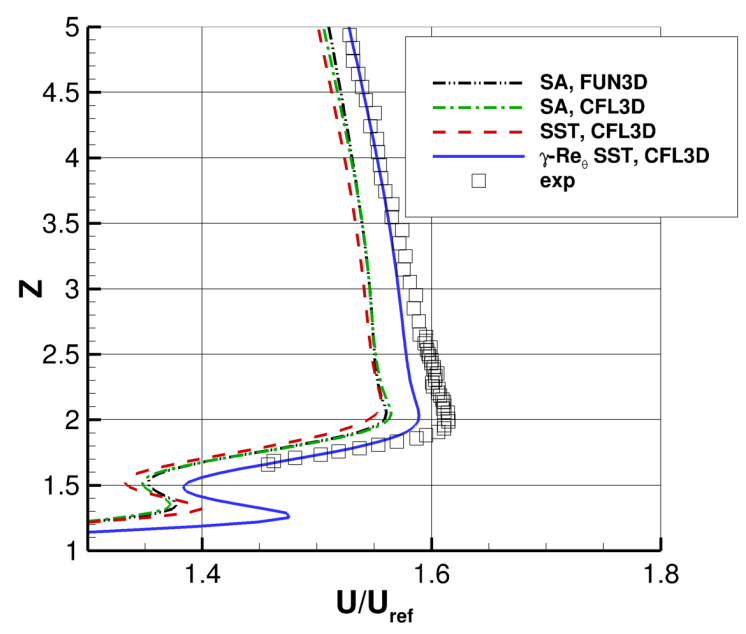

(b) Main element, $\mathbf{8 3 \%}$ span location, near $x=64.2$ inches.

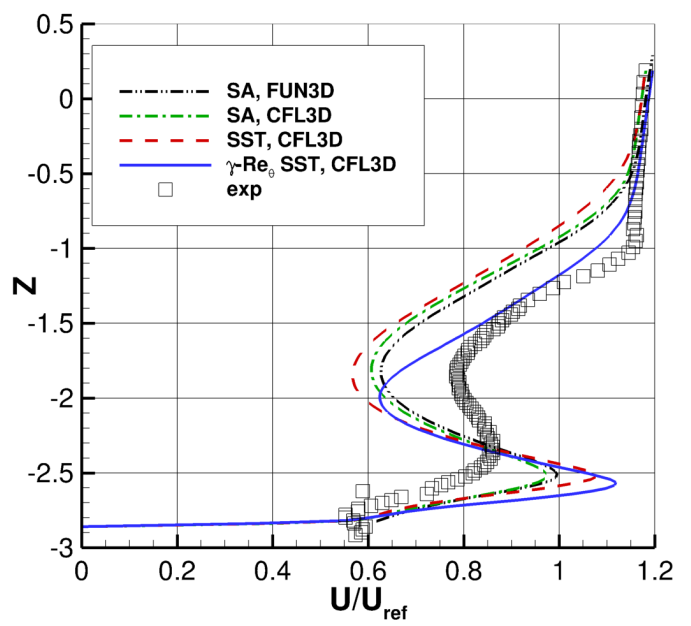

(d) Rear location on flap element, $83 \%$ span location, near $x=$ 75.6 inches.

Figure 7: Comparison of velocity profiles at $\alpha=28^{\circ}$ using different turbulence models, on SX1/UX9 grid (no brackets). 


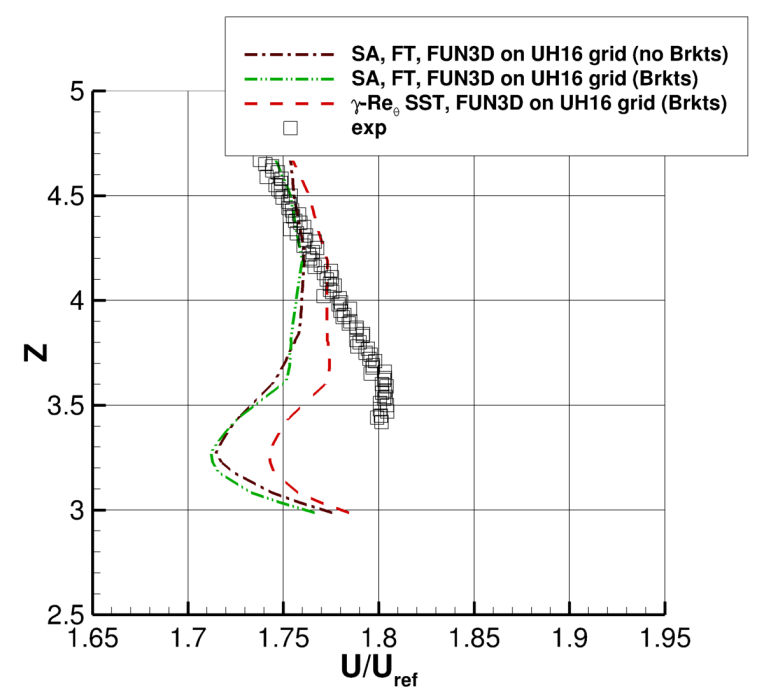

(a) Main element, 15\% span location, near $x=17.5$ inches.

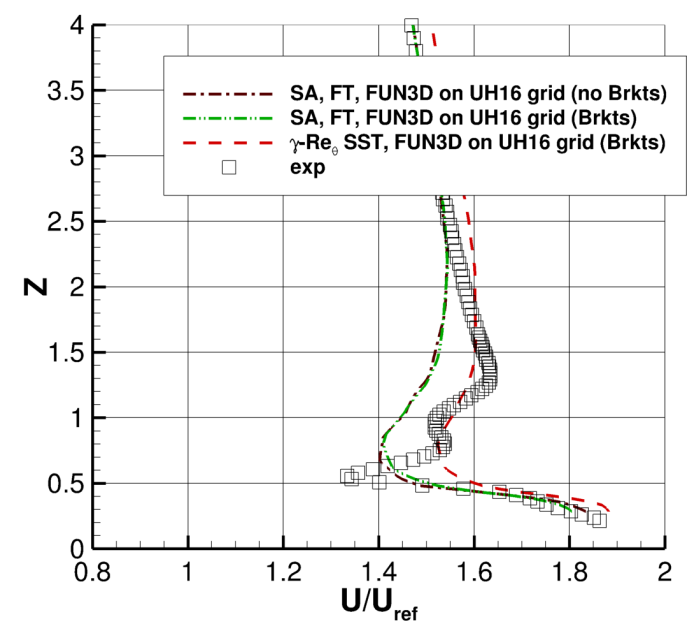

(c) Forward location on flap element, $83 \%$ span location, near $x=69.7$ inches.

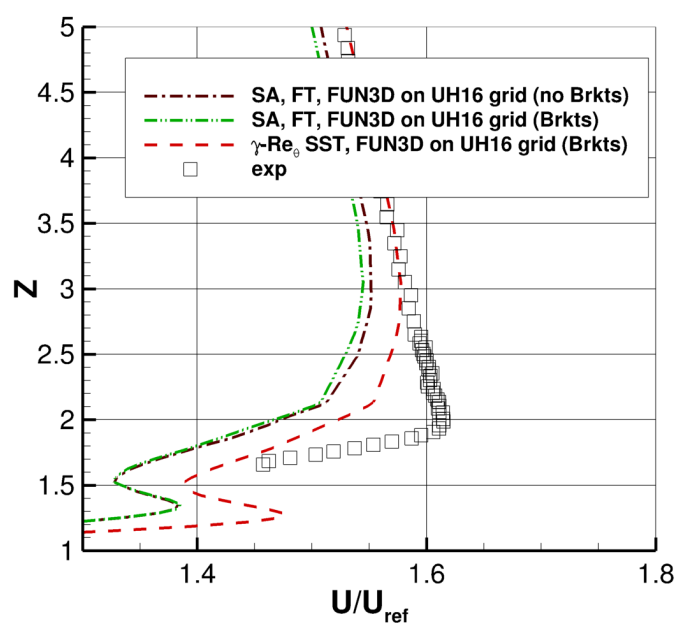

(b) Main element, $\mathbf{8 3} \%$ span location, near $x=64.2$ inches.

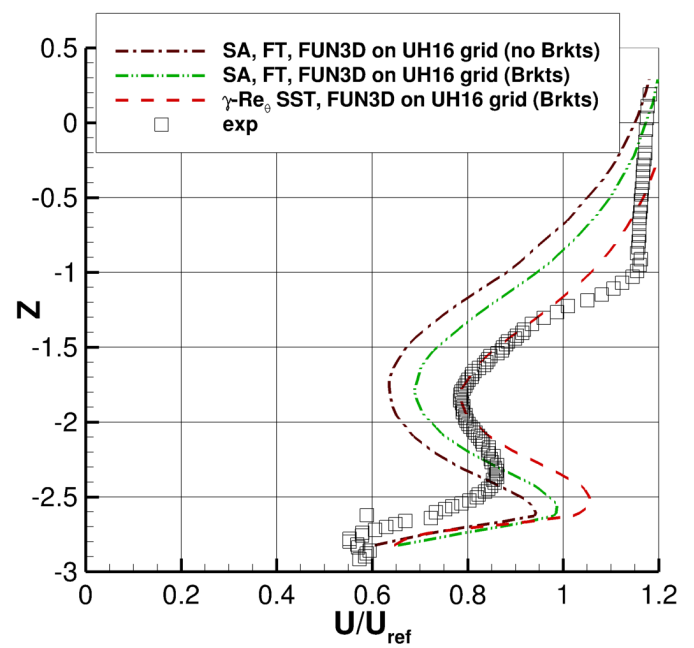

(d) Rear location on flap element, $83 \%$ span location, near $x=$ 75.6 inches.

Figure 8: Comparison of velocity profiles at $\alpha=28^{\circ}$ using different turbulence models, on UH16 grid (both without and with brackets). 


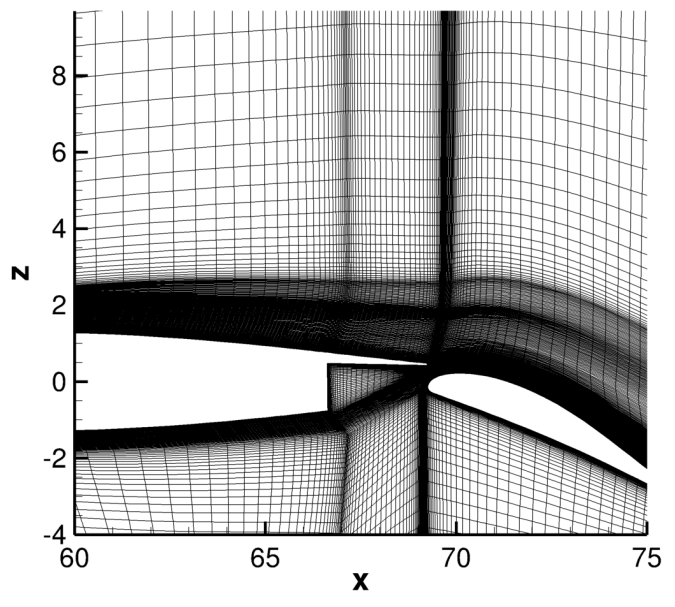

(a) SX1 structured grid.

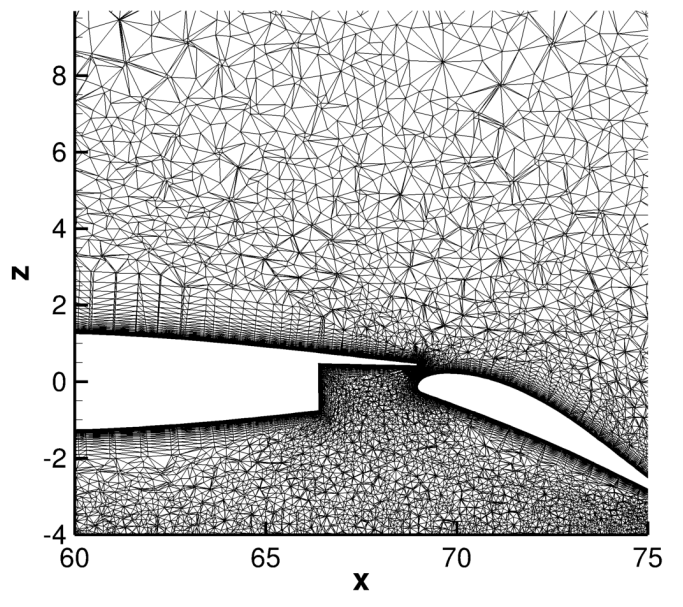

(b) UH16 unstructured grid.

Figure 9: Grid section cuts at $y=-73$ inches (near $85 \%$ span).

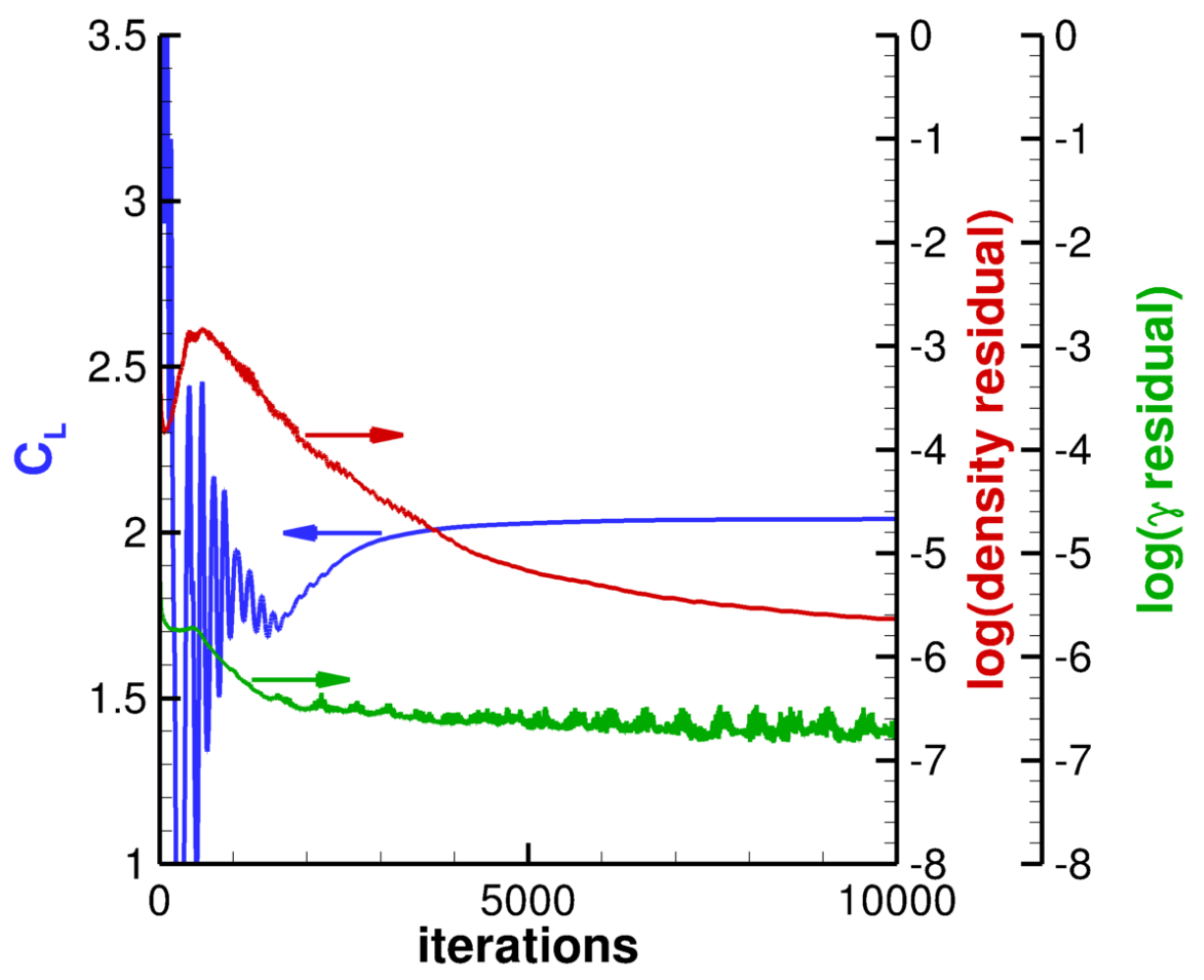

Figure 10: Residual and lift coefficient histories for $\alpha=13^{\circ}$ case, using CFL3D with $\gamma-\operatorname{Re}_{\theta}$ SST model. 


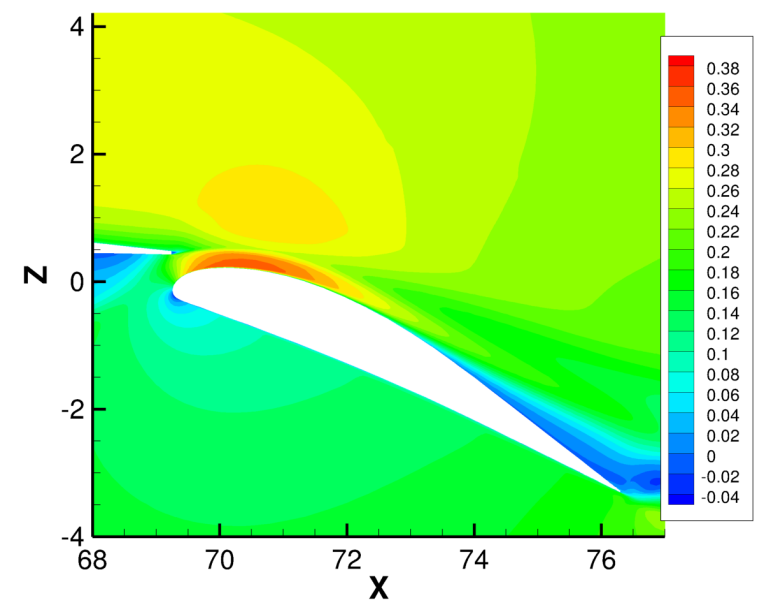

(a) Nondimensional $u$-velocity contours at $y=-73$ inches (near 85\% span), SST model.

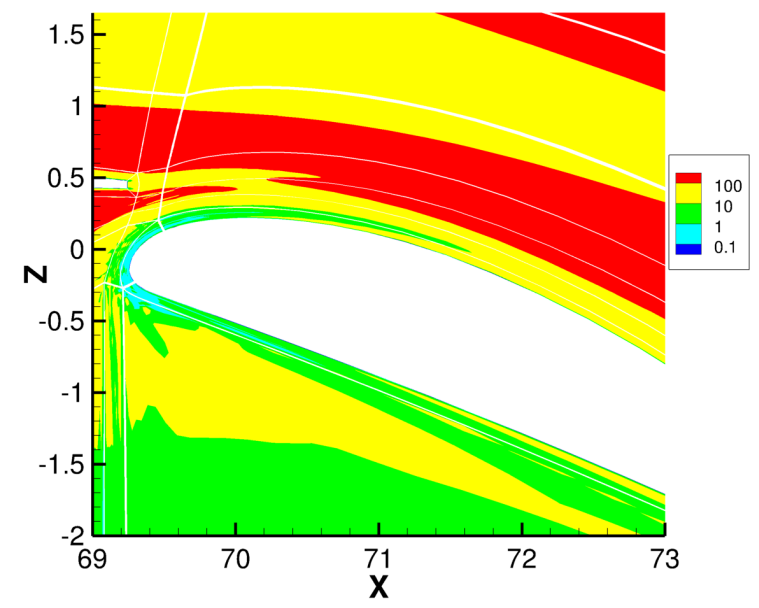

(c) Contours of $\mu_{t} / \mu_{r e f}$ at $y=-73$ inches (color map adjusted to make laminar regions clearly visible), SST model.

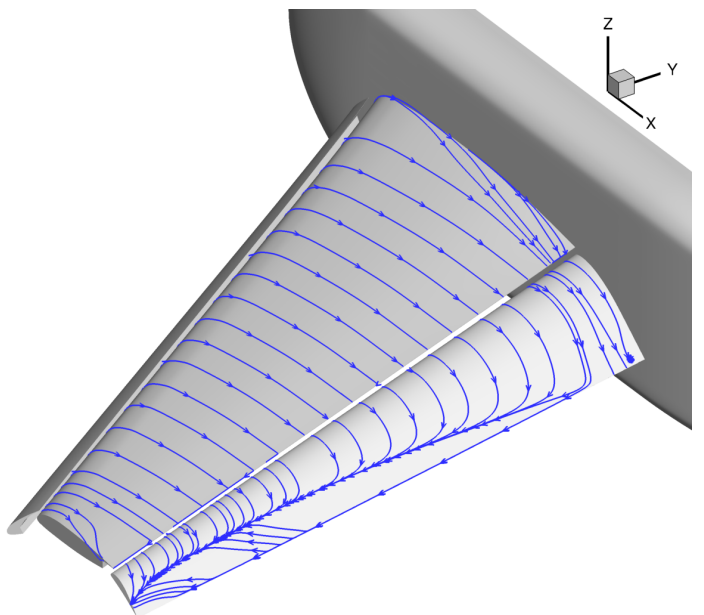

(e) Upper surface streamlines, SST model.

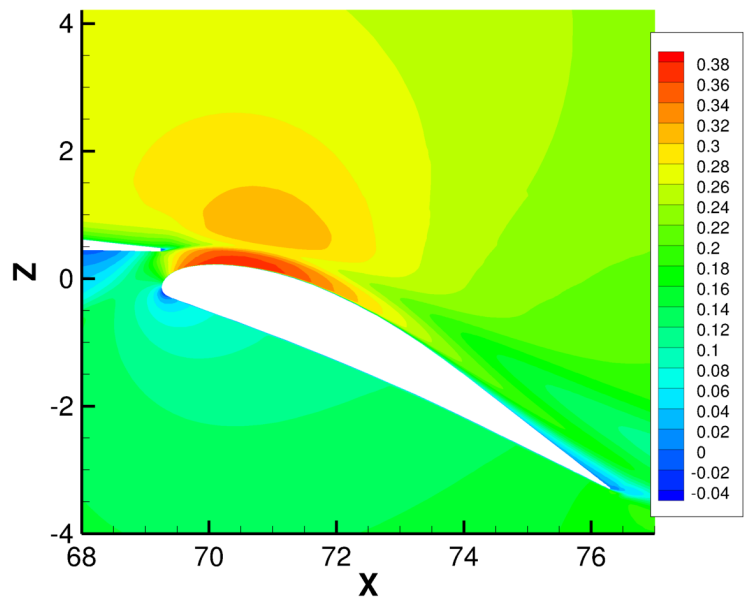

(b) Nondimensional $u$-velocity contours at $y=-73$ inches (near 85\% span), $\gamma-R e_{\theta}$ SST model.

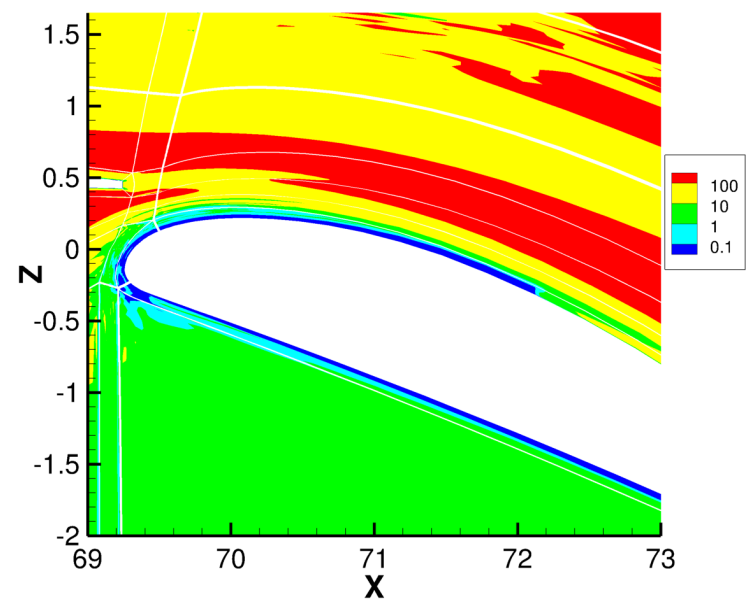

(d) Contours of $\mu_{t} / \mu_{r e f}$ at $y=-73$ inches (color map adjusted to make laminar regions clearly visible), $\gamma-R e_{\theta}$ SST model.

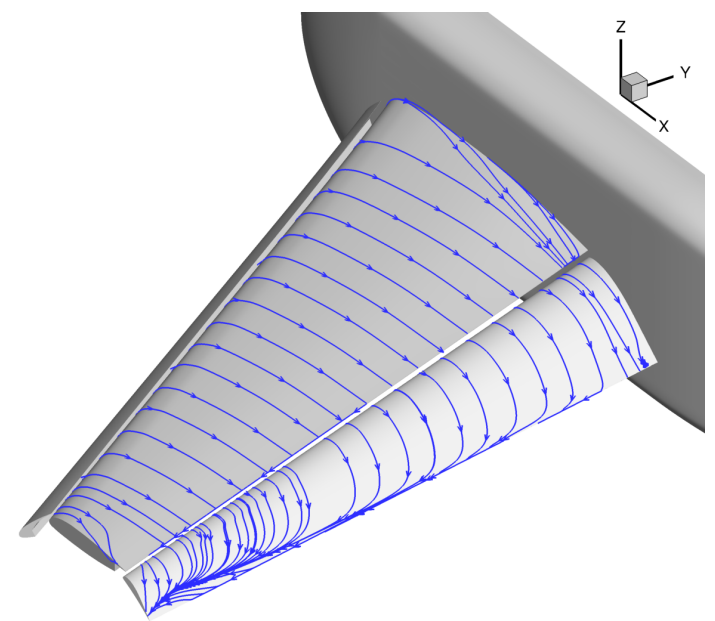

(f) Upper surface streamlines, $\gamma-R e_{\theta}$ SST model.

Figure 11: Comparison of SST model results with $\gamma-R e_{\theta} \operatorname{SST}$ model results, $\alpha=13^{\circ}$ case using CFL3D on SX1 grid. 


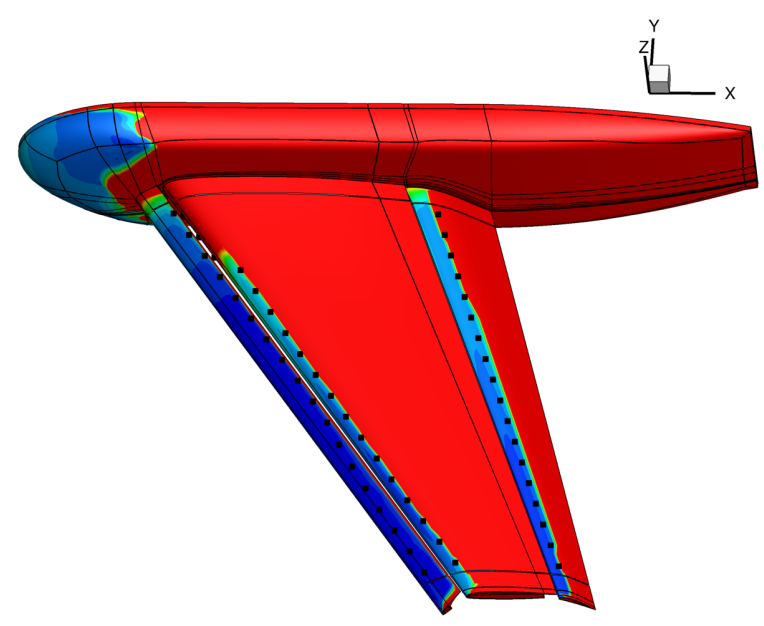

(a) $\alpha=13^{\circ}$, upper surface.

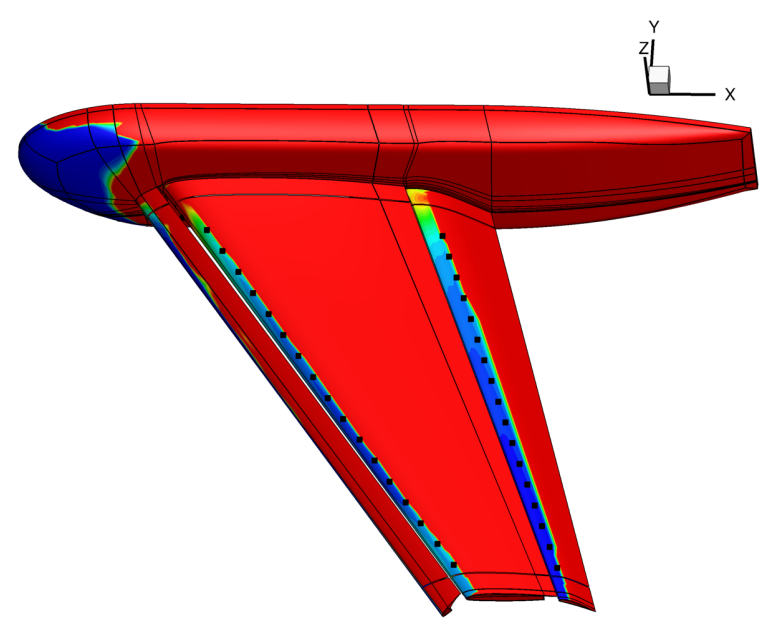

(c) $\alpha=28^{\circ}$, upper surface.

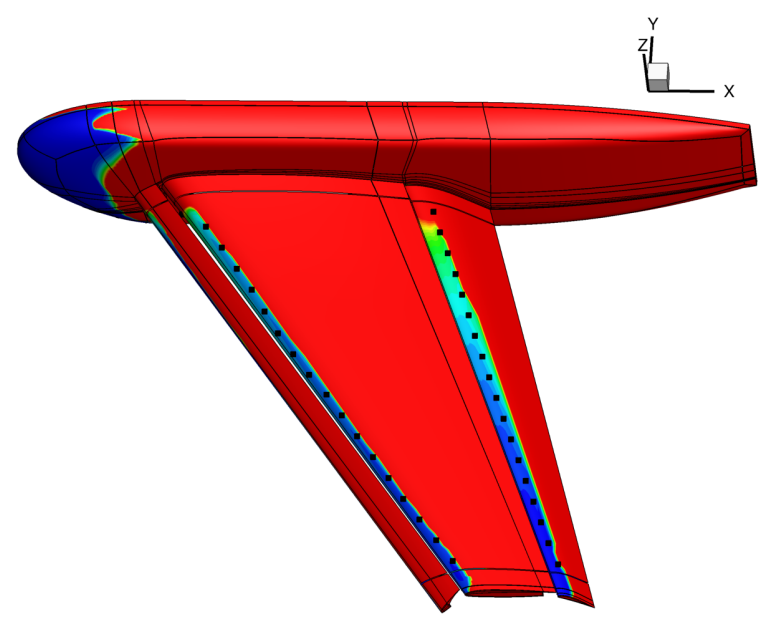

(e) $\alpha=34^{\circ}$, upper surface.

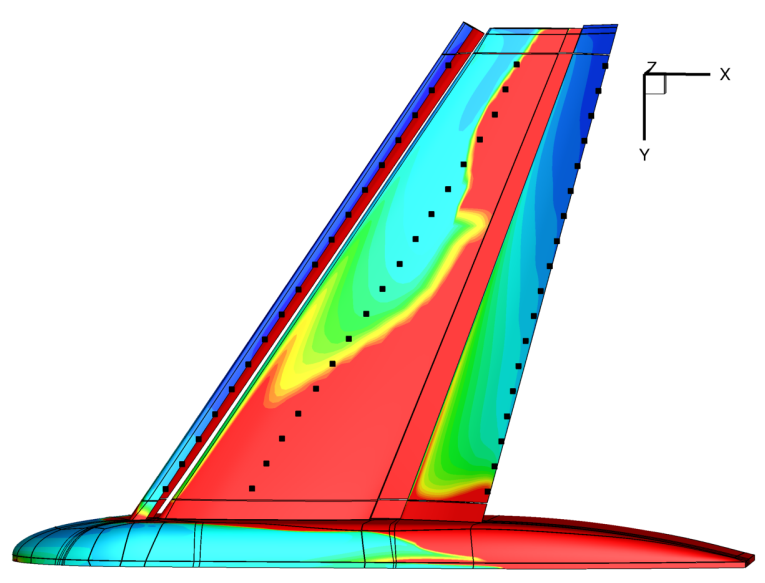

(b) $\alpha=13^{\circ}$, lower surface.

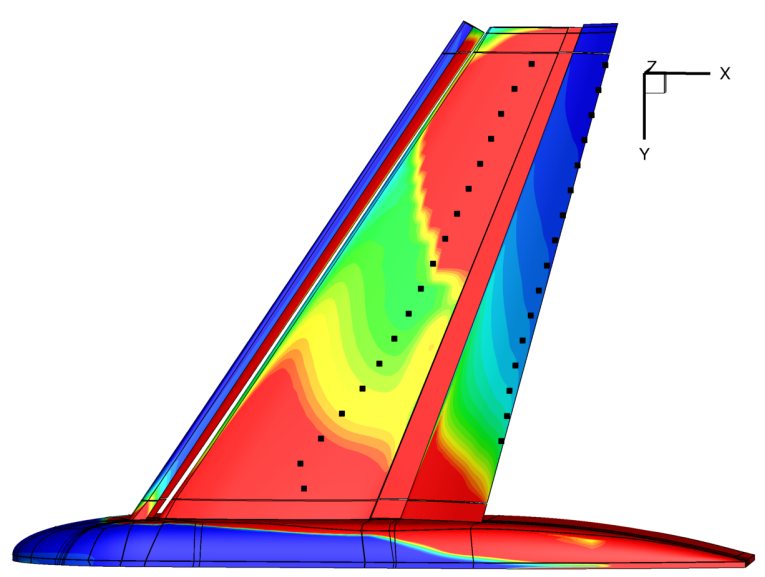

(d) $\alpha=28^{\circ}$, lower surface.

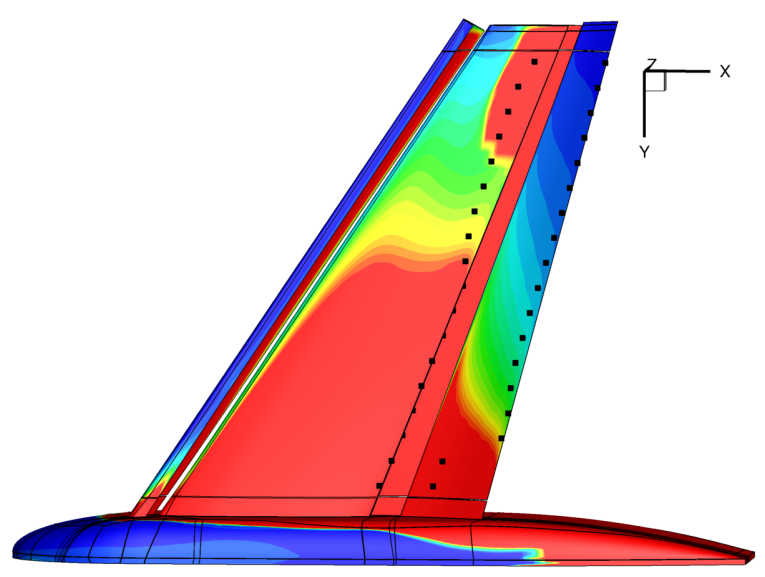

(f) $\alpha=34^{\circ}$, lower surface.

Figure 12: Comparison of approximate transition location predictions; colors represent results from $\gamma-\operatorname{Re}_{\theta} \mathrm{SST}$ model using CFL3D on SX1 grid (Blue=laminar, Red=turbulent); symbols represent results from Eliasson et al. ${ }^{17}$ using $e^{N}$ method (black lines on surface represent zone boundaries). 


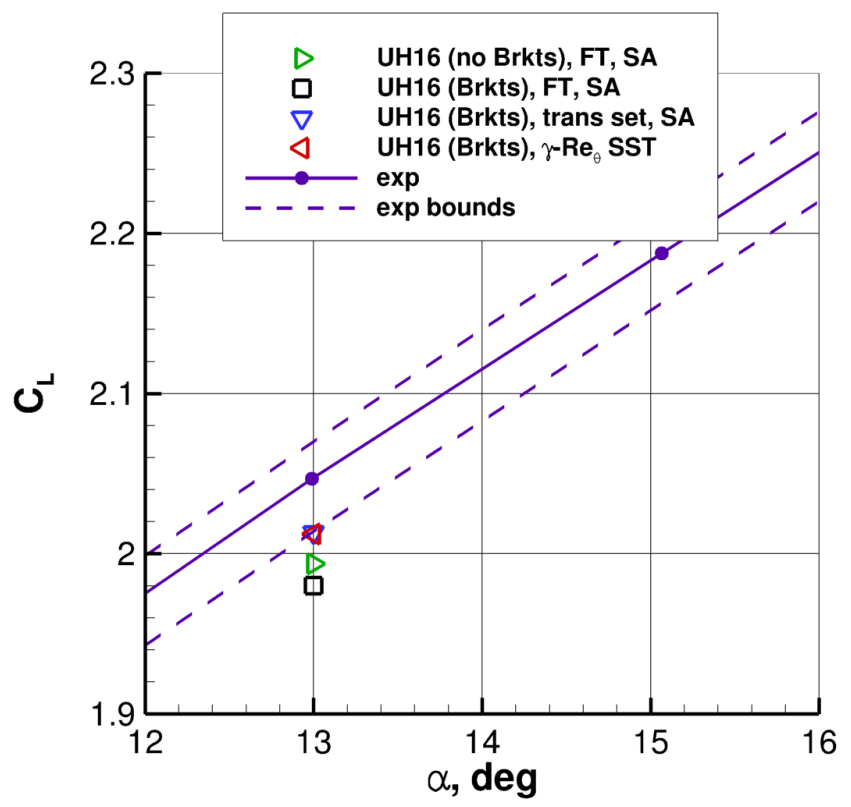

Figure 13: Effects of brackets and transition on $C_{L}$ at $\alpha=13^{\circ}$, using FUN3D on UH16 grid.

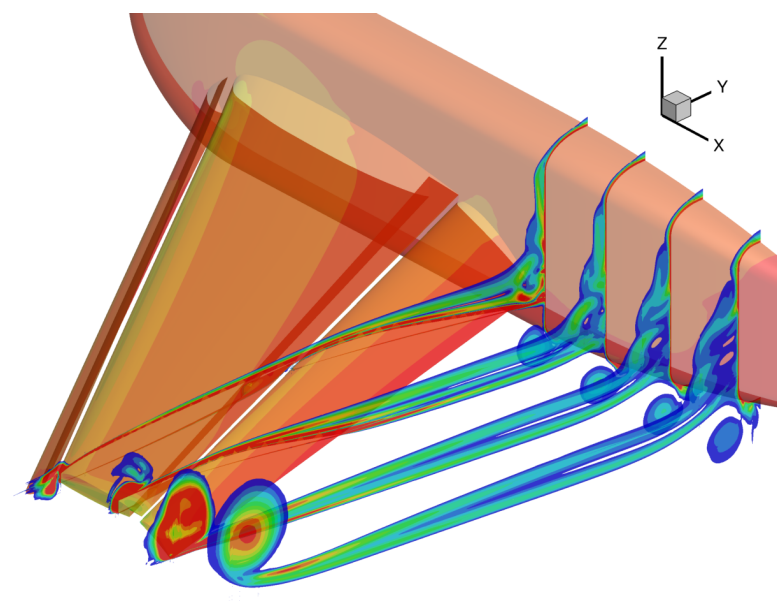

(a) Representative plot for configuration with no brackets.

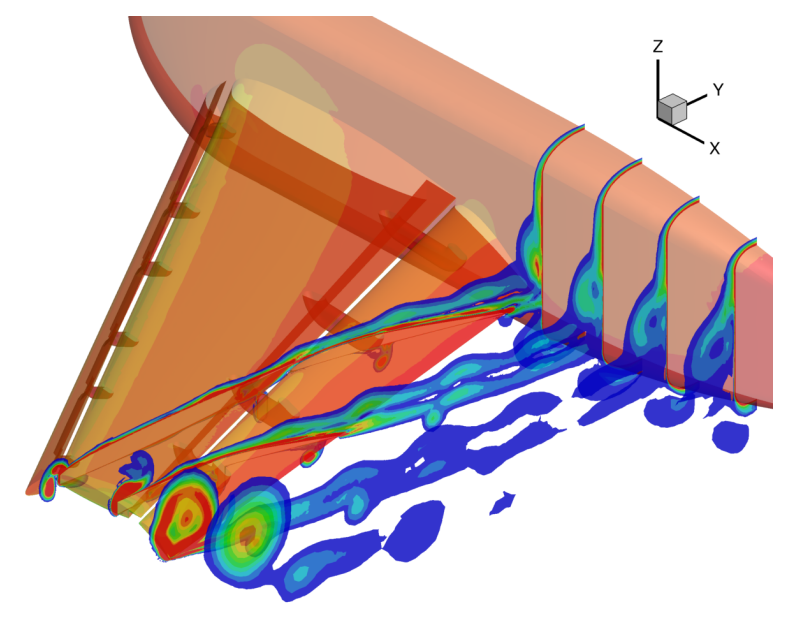

(b) Representative plot for configuration with brackets.

Figure 14: Vorticity contours at $x=60,70,80$, and 90 inches, $\alpha=13^{\circ}$. 


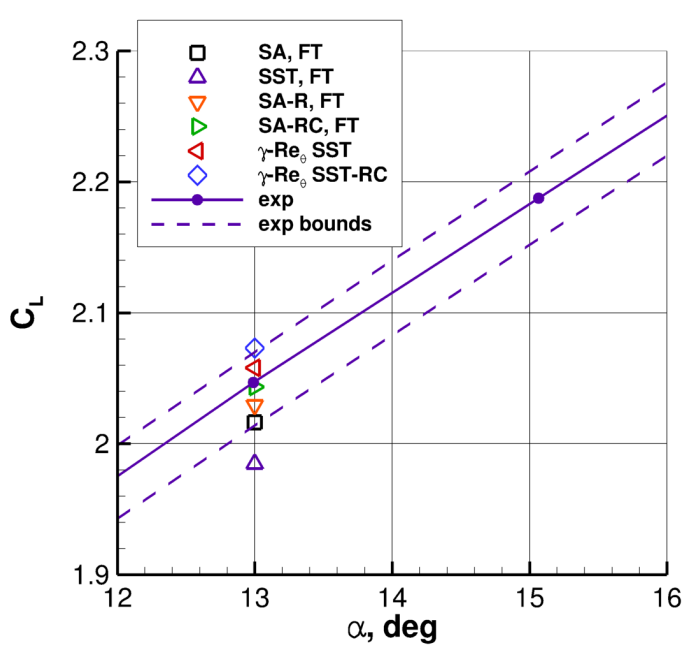

(a) $C_{L}$ at $\alpha=13^{\circ}$, using CFL3D on SX1 grid.

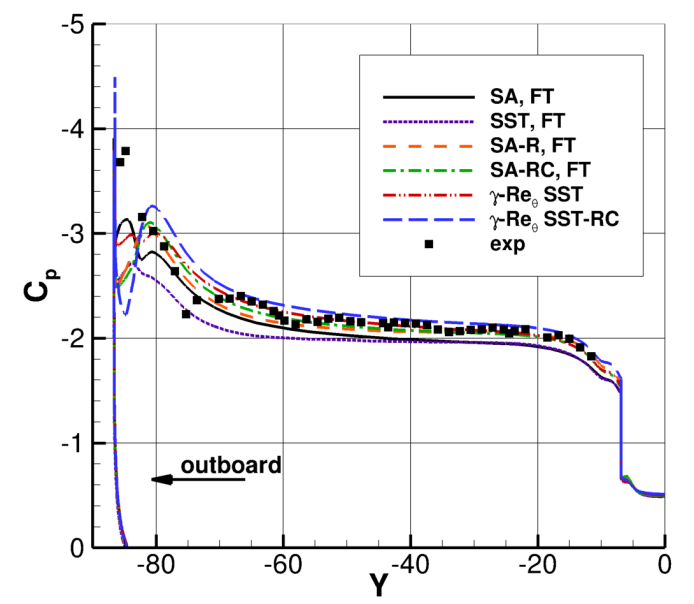

(c) Flap forward $C_{p}$ at $\alpha=13^{\circ}$, using CFL3D on SX1 grid.

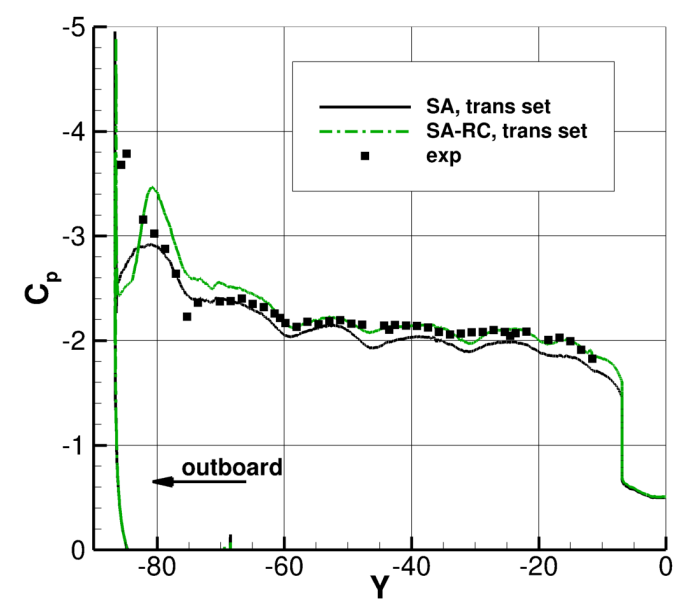

(e) Flap forward $C_{p}$ at $\alpha=13^{\circ}$, using FUN3D (trans set) on UH16 grid with brackets.

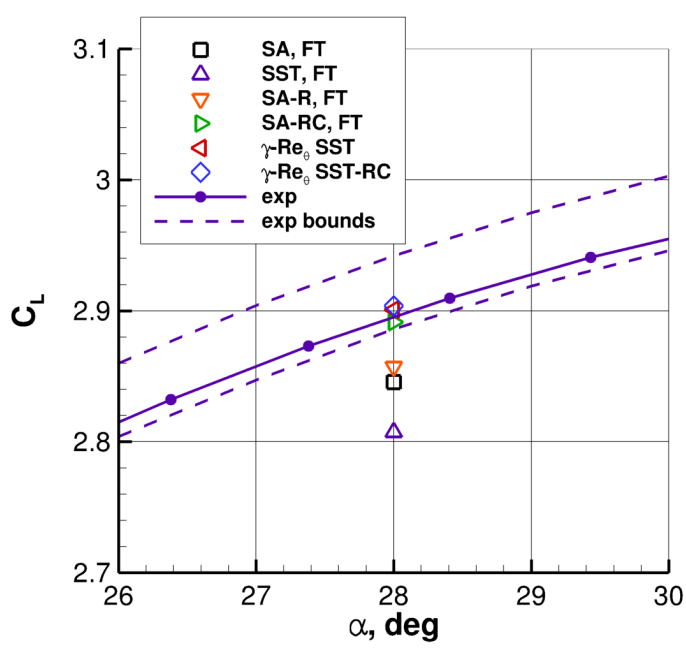

(b) $C_{L}$ at $\alpha=28^{\circ}$, using CFL3D on SX1 grid.

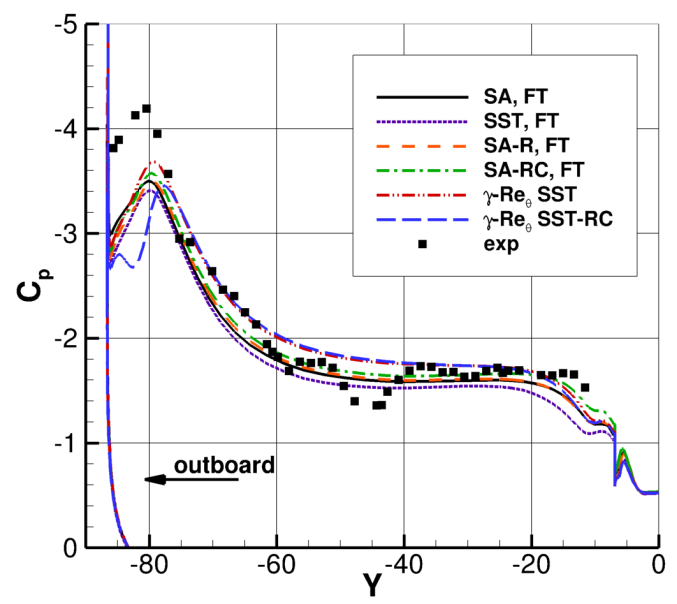

(d) Flap forward $C_{p}$ at $\alpha=28^{\circ}$, using CFL3D on SX1 grid.

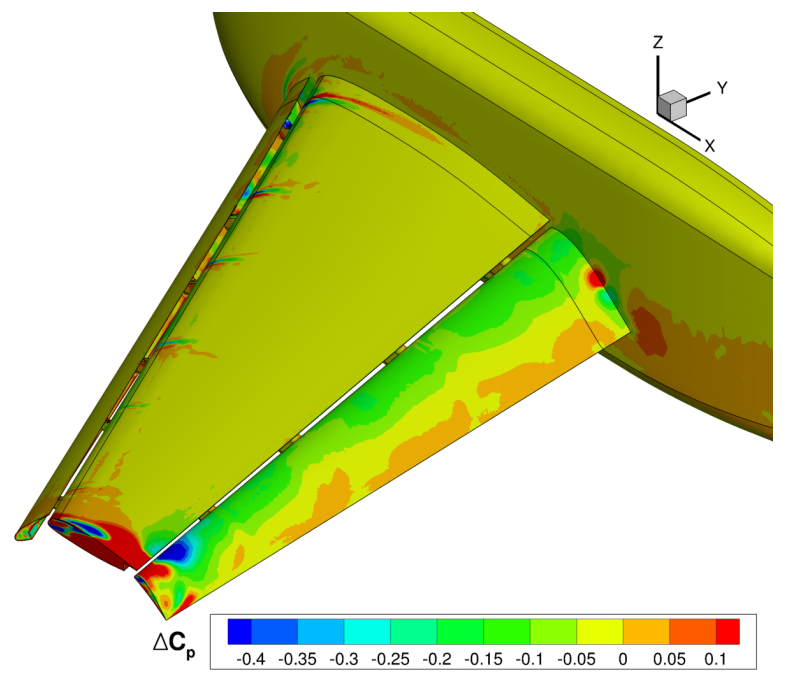

(f) $\Delta C_{p}$ between SA-RC and SA at $\alpha=13^{\circ}$, using FUN3D (trans set) on UH16 grid with brackets.

Figure 15: Effects of various turbulence modeling corrections. 